DRAFT VERSION JULY 12, 2018

Typeset using LATEX twocolumn style in AASTeX61

\title{
A MULTILINE STUDY OF A HIGH-MASS YOUNG STELLAR OBJECT IN THE SMALL MAGELLANIC CLOUD WITH ALMA: THE DETECTION OF METHANOL GAS AT 0.2 SOLAR METALLICITY
}

\author{
Takashi Shimonishi, ${ }^{1,2}$ Yoshimasa Watanabe, ${ }^{3,4}$ Yuri Nishimura,,${ }^{5,6}$ Yuri Aikawa, ${ }^{7}$ Satoshi Yamamoto, ${ }^{8,9}$ Takashi Onaka, ${ }^{7}$ Nami Sakai, ${ }^{10}$ and \\ Aкiko Kawamura ${ }^{6}$ \\ ${ }^{1}$ Frontier Research Institute for Interdisciplinary Sciences, Tohoku University, Aramakiazaaoba 6-3, Aoba-ku, Sendai, Miyagi, 980-8578, Japan \\ ${ }^{2}$ Astronomical Institute, Tohoku University, Aramakiazaaoba 6-3, Aoba-ku, Sendai, Miyagi, 980-8578, Japan \\ ${ }^{3}$ Faculty of Pure and Applied Sciences, University of Tsukuba, 1-1-1, Tennodai, Tsukuba, Ibaraki 305-8577, Japan \\ ${ }^{4}$ Tomonaga Center for the History of the Universe, University of Tsukuba, Tsukuba, Ibaraki 305-8571, Japan \\ ${ }^{5}$ Institute of Astronomy, The University of Tokyo, 2-21-1, Osawa, Mitaka, Tokyo 181-0015, Japan \\ ${ }^{6}$ National Astronomical Observatory of Japan, 2-21-1, Osawa, Mitaka, Tokyo 181-8588, Japan \\ ${ }^{7}$ Department of Astronomy, Graduate School of Science, The University of Tokyo, 7-3-1 Hongo, Bunkyo-ku, Tokyo 113-0033, Japan \\ ${ }^{8}$ Department of Physics, The University of Tokyo, 7-3-1, Hongo, Bunkyo-ku, Tokyo 113-0033, Japan \\ ${ }^{9}$ Research Center for the Early Universe, The University of Tokyo, 7-3-1, Hongo, Bunkyo-ku, Tokyo, 113-0033, Japan \\ ${ }^{10}$ RIKEN, 2-1 Hirosawa, Wako, Saitama 351-0198, Japan
}

\begin{abstract}
We report the results of subparsec-scale submillimeter observations towards an embedded high-mass young stellar object in the Small Magellanic Cloud (SMC) with ALMA. Complementary infrared data obtained with the AKARI satellite and the Gemini South telescope are also presented. The target infrared point source is spatially resolved into two dense molecular cloud cores; one is associated with a high-mass young stellar object (YSO core), while another is not associated with an infrared source (East core). The two cores are dynamically associated but show different chemical characteristics. Emission lines of $\mathrm{CS}, \mathrm{C}^{33} \mathrm{~S}, \mathrm{H}_{2} \mathrm{CS}$, $\mathrm{SO}, \mathrm{SO}_{2}, \mathrm{CH}_{3} \mathrm{OH}, \mathrm{H}^{13} \mathrm{CO}^{+}, \mathrm{H}^{13} \mathrm{CN}, \mathrm{SiO}$, and dust continuum are detected from the observed region. Tentative detection of HDS is also reported. The first detection of $\mathrm{CH}_{3} \mathrm{OH}$ in the $\mathrm{SMC}$ has a strong impact on our understanding of the formation of complex organic molecules in metal-poor environments. The gas temperature is estimated to be $\sim 10 \mathrm{~K}$ based on the rotation analysis of $\mathrm{CH}_{3} \mathrm{OH}$ lines. The fractional abundance of $\mathrm{CH}_{3} \mathrm{OH}$ gas in the East core is estimated to be $(0.5-1.5) \times 10^{-8}$, which is comparable with or marginally higher than those of similar cold sources in our Galaxy despite a factor of five lower metallicity in the SMC. This work provides observational evidence that an organic molecule like $\mathrm{CH}_{3} \mathrm{OH}$, which is largely formed on grain surfaces, can be produced even in a significantly lower metallicity environment compared to the solar neighborhood. A possible origin of cold $\mathrm{CH}_{3} \mathrm{OH}$ gas in the observed dense core is discussed.
\end{abstract}

Keywords: astrochemistry - Magellanic Clouds - ISM: abundances - ISM: molecules - circumstellar matter radio lines: ISM - infrared: ISM 


\section{INTRODUCTION}

Interstellar chemistry at low metallicity is crucial to understand chemical processes in past galaxies since cosmic metallicity is increasing in time with the evolution of our universe. Observational studies of dense molecular gas in nearby lowmetallicity galaxies play an important role in understanding chemistry in star-forming regions as a function of galactic metallicity. Chemical compositions of molecular cloud cores and protostellar envelopes are particularly interesting for understanding initial chemical conditions of star-formation in metal-poor environments.

Characteristic chemical compositions around high-mass young stellar objects (YSOs) in low metallicity environments are emerging thanks to recent advances in infrared and radio observations towards compact sources in the Large Magellanic Cloud. The abundance of organic molecules such as $\mathrm{CH}_{3} \mathrm{OH}$ is one of the key issues in recent astrochemical studies toward low metallicity systems. Infrared observations of ices suggest that solid $\mathrm{CH}_{3} \mathrm{OH}$ around embedded high-mass YSOs in the LMC is less abundant than those of Galactic similar objects (Shimonishi et al. 2016a). In single-dish radio observations, $\mathrm{CH}_{3} \mathrm{OH}$ lines are detected from two LMC star-forming regions (Heikkilä et al. 1999; Wang et al. 2009). Spectral line surveys and abundance analyses towards seven molecular clouds in the LMC suggest that $\mathrm{CH}_{3} \mathrm{OH}$ gas is less abundant in the LMC (Nishimura et al. 2016a). Searches for maser emission in star-forming regions reported the lower detection rate of of $\mathrm{CH}_{3} \mathrm{OH}$ masers in the LMC compared to those in our Galaxy (e.g., Green et al. 2008; Ellingsen et al. 2010). Submillimeter observations with the Atacama Large Millimeter/submillimeter Array (ALMA) reported that gasphase $\mathrm{CH}_{3} \mathrm{OH}$ towards a hot molecular core in the LMC is underabundant by 1-3 orders of magnitude compared to those towards Galactic hot cores (Shimonishi et al. 2016b). On the other hand, Sewiło et al. (2018) recently detected $\mathrm{CH}_{3} \mathrm{OH}$ and other complex organic molecules toward two different hot molecular cores in the LMC. They argue that the abundances of these molecules are scaled by the metallicity of the LMC and comparable to those found at the lower end of the range in Galactic hot cores. Chemical processes of organic molecules in low metallicity environments are still an open question and observational tests for further metal-poor samples are essentially important.

The Small Magellanic Cloud (SMC), the prime target of this paper, is one of the nearest star-forming galaxies to our Galaxy (62.1 $\pm 2.0 \mathrm{kpc}$, Graczyk et al. 2014). It is well known that the SMC has a low-metallicity environment, which is about one-fifth of the solar neighborhood (e.g., Dufour 1984; Russell \& Dopita 1992; Venn 1999). Relative abundances among heavy elements are also different in the SMC, e.g., the ratio of elemental nitrogen relative to carbon or oxygen is lower in the SMC by a factor of $\sim 3$
(Russell \& Dopita 1992). A dust-to-gas ratio is also reported to be lower in the SMC than in our Galaxy by a factor of 5 or more (e.g., Leroy et al. 2007; Roman-Duval et al. 2017). A lower abundance of dust grains leads to harsh radiation environment, which results in elevated dust/gas temperatures and more effective photoprocessing of dense interstellar medium (e.g., Aguirre et al. 2003; van Loon et al. 2010). The above environmental differences would affect chemical compositions in molecular clouds in the SMC as in the case of the LMC.

Pioneering works on molecular gas multiline studies for the SMC are presented in Chin et al. (1997, 1998) and Heikkilä et al. (1999) based on observations with the 15-m Swedish-ESO Submillimetre Telescope. These studies reported detection of various molecular species, including $\mathrm{CO}$, $\mathrm{CS}, \mathrm{SO}, \mathrm{C}_{2} \mathrm{H}, \mathrm{HCO}^{+}, \mathrm{HCN}, \mathrm{HNC}, \mathrm{H}_{2} \mathrm{CO}$, and $\mathrm{C}_{3} \mathrm{H}_{2}$ towards active star-forming regions $\mathrm{N} 27$ and LIRS 36. Recently, Muraoka et al. (2017) reported high-spatial resolution observations of $\mathrm{CO},{ }^{13} \mathrm{CO}, \mathrm{C}^{18} \mathrm{O}$, and $\mathrm{CS}$ with ALMA towards a star-forming region, N83C. They revealed subparsec-scale structures and physical properties of molecular clouds in the SMC. High spatial resolution molecular line observations towards the SMC are, however, still limited to a few molecular species, and the chemistry of star-forming cores in the SMC remains to be investigated.

Infrared observations of ices toward embedded highmass YSOs in the SMC are also reported in the literature (van Loon et al. 2008; Oliveira et al. 2011, 2013). Currently, $\mathrm{H}_{2} \mathrm{O}$ and $\mathrm{CO}_{2}$ ices are detected in the SMC, while $\mathrm{CO}$ ice is not detected. Astrochemical simulations dedicated to the SMC environment suggest that the observed absence of $\mathrm{CO}$ ice in the SMC is explained by a combined effect of increased dust temperatures and reduced metal abundances (Acharyya \& Herbst 2016; Pauly \& Garrod 2018).

In this paper, we report the results of subparsec-resolution submillimeter observations towards a high-mass YSO in the SMC with ALMA. Section 2 describes the details of observations and data reduction. The obtained spectra and images are presented in Section 3. Analysis of the spectral data and the derivation of the physical quantities of molecular gas are also described in this section. Section 4 describes physical and chemical properties of the observed source. A particular focus is given to the properties of $\mathrm{CH}_{3} \mathrm{OH}$ gas in the metalpoor environment of the SMC. The conclusions of this paper are presented in Section 5.

\section{OBSERVATIONS AND DATA REDUCTION}

\subsection{Target}

The target is the infrared source IRAS 01042-7215 located in the SMC. It is spectroscopically identified to be an embedded high-mass YSO in previous infrared studies (van Loon et al. 2008; Oliveira et al. 2011, 2013; Shimonishi 
2012; Ruffle et al. 2015). The luminosity of the source is estimated to be $2 \times 10^{4} \mathrm{~L}_{\odot}$. More detailed discussion about the YSO's properties is given in Section 4.

\subsection{Observations}

Observations were carried out with ALMA in November 2016 as a part of the Cycle 4 program 2016.1.00394.S (PI T. Shimonishi). The telescopes were pointed to RA = $01^{\mathrm{h}} 05^{\mathrm{m}} 49.32$ and Dec $=-71^{\circ} 59^{\prime} 48^{\prime \prime} 4$ (J2000), which corresponds to the target's infrared peak position. The target was observed in Band 6 with four spectral windows covering 241.48-243.36, 243.84-245.71, 256.99-258.87, and 258.85$260.72 \mathrm{GHz}$ in the rest-frame frequency. The frequency resolution is $977 \mathrm{kHz}$ before channel averaging. The total onsource integration time is 39 minutes. We use J2357-5311, J2056-4714, and J0102-7546 as a flux, bandpass, and gain calibrator, respectively. In total 43 antennas are used for the observations with the minimum and maximum baseline length of $19 \mathrm{~m}$ and $1.1 \mathrm{~km}$.

\subsection{Data reduction}

Raw interferometric data are processed using the Common Astronomy Software Applications (CASA) package. The calibration is done with CASA 4.7.0 and the imaging is with CASA 4.7.2. The synthesized beam size is $0.35-0.37^{\prime \prime} \times$ $0.22-0.23^{\prime \prime}$ with a position angle of -25 degree, which corresponds to $0.11 \mathrm{pc} \times 0.07 \mathrm{pc}$ at the distance of the SMC (62.1 $\mathrm{kpc})$. The primary beam has a full-width at half-maximum (FWHM) of about 25", and the maximum recoverable angular scale is about $3^{\prime \prime}$. The primary beam correction is done by using the impbcor task in CASA.

The spectra and continuum flux are extracted from the $0.34^{\prime \prime}(0.10 \mathrm{pc})$ diameter region centered at the three positions (Position 1, 2, 3, hereafter P1, P2, P3), where the dust continuum and/or molecular emission lines have a local peak (see Section 3.2). Their coordinates are listed in Table 1. The continuum emission is subtracted from the spectral data using the uvcontsub task in CASA. The velocity resolution of the extracted spectra is $1.5 \mathrm{~km} \mathrm{~s}^{-1}$.

\subsection{Complementary infrared data}

Complementary infrared datasets are also presented in this work. One contains high spatial resolution mid-infrared imaging data obtained with T-ReCS at the Gemini South telescope (Program ID: S10B-120, PI: T. Shimonishi). The observations were carried out using a broad-band filter in the N-band (7.70-12.97 $\mu \mathrm{m}$, centered at $10.36 \mu \mathrm{m})$ as well as six narrow-band filters (centered at 7.73, 8.74, 9,69, 10.38, 11.66 , and $12.33 \mu \mathrm{m}$ ). The spatial resolution of the imaging data is about $0.55^{\prime \prime}$ according to the FWHM measurement for a photometric standard star.

Another complementary data set is a near-infrared 2-5 $\mu \mathrm{m}$ spectrum obtained with the Infrared Camera (IRC) on board AKARI (Murakami et al. 2007; Onaka et al. 2007). The observations were carried out using the slitless NG spectroscopy mode with the Np point source aperture $(\mathrm{R} \sim 80)$ as a part of the $A K A R I$ post-helium mission program (Program ID: LMCNG, PI: T. Onaka). The near-infrared spectrum is extracted from the infrared peak position. A typical FWHM of point sources in the AKARI data is approximately $5^{\prime \prime}-8^{\prime \prime}$.

\section{RESULTS}

\subsection{Spectra}

Figures 1-3 show spectra extracted from the three positions described in Section 2.3. Spectral lines are identified with the aid of the Cologne Database for Molecular Spectroscopy ${ }^{1}$ (CDMS, Müller et al. 2001, 2005). The detection criteria adopted here are the $3 \sigma$ significance level and the velocity coincidence with the systemic velocity of nearby clouds (between $186 \mathrm{~km} \mathrm{~s}^{-1}$ and $196 \mathrm{~km} \mathrm{~s}^{-1}$, Israel et al. 1993). Line parameters are measured by fitting a single Gaussian profile to observed lines. In some cases we subtract a local baseline which is estimated from adjacent line-free regions by fitting a polynomial to correct for small baseline ripples. Based on the fitting, we estimate a peak brightness temperature, an FWHM, an LSR velocity, and an integrated intensity for each line. For $\mathrm{CH}_{3} \mathrm{OH}\left(5_{0}-4_{0} \mathrm{E}\right)$ and $\left(5_{ \pm 2}-4_{ \pm 2}\right.$ E) lines, the integrated intensities are calculated by directly integrating the spectrum between $190 \mathrm{~km} \mathrm{~s}^{-1}$ and $194 \mathrm{~km}$ $\mathrm{s}^{-1}$ because they are too narrow to fit a Gaussian. These two lines are attributed to tentative detections given their low intensity. The results of the spectral fits are shown in Figures $1-3$, and the measured line parameters are summarized in Table 2. The table also contains the estimated upper limits on several important non-detection lines.

Molecular emission lines of $\mathrm{CS}, \mathrm{C}^{33} \mathrm{~S}, \mathrm{H}_{2} \mathrm{CS}, \mathrm{SO}, \mathrm{SO}_{2}$, $\mathrm{CH}_{3} \mathrm{OH}, \mathrm{H}^{13} \mathrm{CO}^{+}, \mathrm{H}^{13} \mathrm{CN}$, and $\mathrm{SiO}$ are detected from the observed region. Among these species, $\mathrm{CH}_{3} \mathrm{OH}, \mathrm{SO}_{2}, \mathrm{H}_{2} \mathrm{CS}$, $\mathrm{C}^{33} \mathrm{~S}$, and $\mathrm{H}^{13} \mathrm{CN}$ are for the first time detected in the SMC. Measured line widths are typically $3-4 \mathrm{~km} \mathrm{~s}^{-1}$. The spectra at P1 show slightly broader line widths compared to those at P2 and P3. An unidentified line is also detected near 244.5556 $\mathrm{GHz}$ (hereafter U244.556), which is tentatively attributed to HDS as further discussed in Section 4.4. High-excitation lines (upper-state energy $>100 \mathrm{~K}$ ) are covered in the present frequency settings but not detected. A carbon-chain-related molecule, $c-\mathrm{C}_{3} \mathrm{H}_{2}$, is not detected.

\subsection{Synthesized images}

Figure 4 shows synthesized images of continuum and molecular emission lines observed toward the target region. The images are constructed by integrating spectral data in

\footnotetext{
${ }^{1}$ https://www.astro.uni-koeln.de/cdms
} 
Table 1. Positions for the spectral extraction

\begin{tabular}{|c|c|c|c|c|}
\hline Position & $\begin{array}{c}\text { R.A. } \\
\text { (J2000) }\end{array}$ & $\begin{array}{l}\text { Decl. } \\
(\mathrm{J} 2000)\end{array}$ & Detected molecules & Note \\
\hline $\mathrm{P} 1$ & 010549.33 & -715948.46 & $\mathrm{CS}, \mathrm{SO}, \mathrm{H}^{13} \mathrm{CO}^{+}$ & Continuum, $\mathrm{H}^{13} \mathrm{CO}^{+}$, and infrared peak \\
\hline P2 & 010549.54 & -715948.94 & 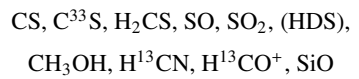 & $\mathrm{C}^{33} \mathrm{~S}, \mathrm{H}_{2} \mathrm{CS}$, and $\mathrm{SiO}$ peak \\
\hline P3 & 010549.50 & -715949.41 & $\mathrm{CS}, \mathrm{SO}, \mathrm{SO}_{2}, \mathrm{CH}_{3} \mathrm{OH}, \mathrm{H}^{13} \mathrm{CO}^{+}$ & $\mathrm{CH}_{3} \mathrm{OH}$ peak \\
\hline
\end{tabular}

Note-Tabulated positions are shown in Figure 4. HDS is a tentative detection.
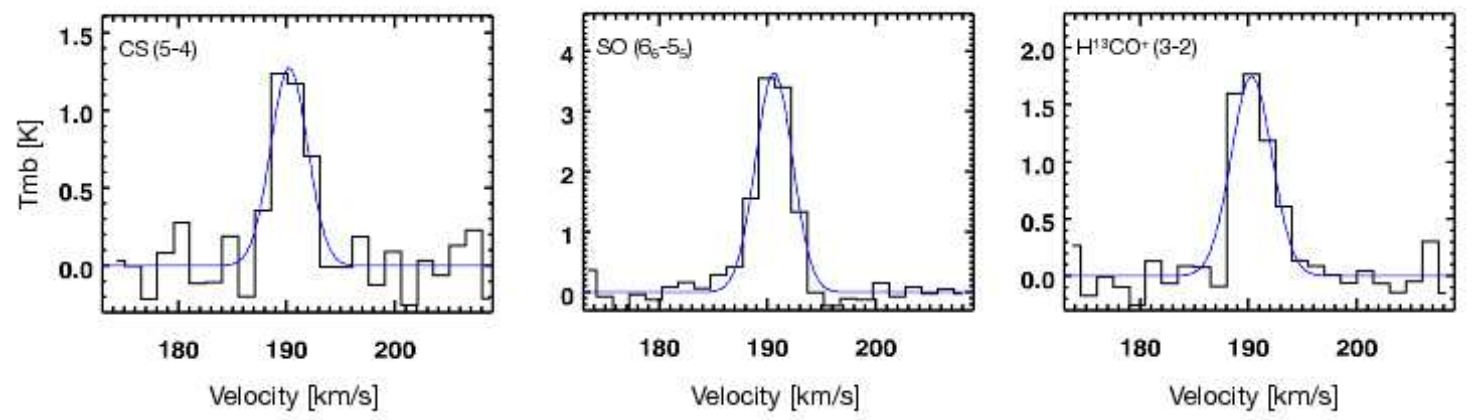

Figure 1. Spectra extracted at Position 1. Emission lines of $\mathrm{CS}, \mathrm{SO}$, and $\mathrm{H}^{13} \mathrm{CO}^{+}$are shown. The blue lines represent Gaussian profiles fitted to the spectra.

the velocity range where the emission is seen. For $\mathrm{CH}_{3} \mathrm{OH}$, two lines with similar spectroscopic parameters $\left(5_{0}-4_{0} \mathrm{~A}^{+}\right.$ and $\left.5_{-1}-4_{-1} \mathrm{E}\right)$ are added together to increase the signal-tonoise ratio $(\mathrm{S} / \mathrm{N})$. The $\mathrm{H}^{13} \mathrm{CN}$ map is not shown here because the line is too weak to derive the distribution. In addition to the submillimeter data, a high spatial resolution mid-infrared $10 \mu \mathrm{m}$ image obtained with Gemini/T-ReCS is shown in the figure.

The distribution of continuum emission shows two peaks; one is associated with an embedded high-mass YSO (P1), and another is located at the east side of the YSO (P2) separated by about $1^{\prime \prime}(0.3 \mathrm{pc})$. The latter is not seen in the mid-infrared image. The near-infrared image presented in Ward et al. (2017) shows compact emission centered around $\mathrm{P} 1$, which is similar to our mid-infrared image. Note that the criterion for the continuum detection is the $3 \sigma$ significance level, and the $\mathrm{S} / \mathrm{Ns}$ at $\mathrm{P} 1, \mathrm{P} 2$, and $\mathrm{P} 3$ are 14,8 , and 3, respectively. It seems that the YSO's position is slightly offset from the center of $\mathrm{P} 1$, but the separation is smaller than half of the beam size, and the positional accuracy of the infrared source is about $0.3^{\prime \prime}$, thus we regard that the YSO and $\mathrm{P} 1$ are spatially coincident within the accuracy of the present dataset. Hereafter, the molecular cloud core at the YSO's position is referred to as the YSO core and the one on the east side as the East core. The size of the continuum emission region (FWHM of a two-dimensional Gaussian fit) is about $0.5-0.6^{\prime \prime}(0.15-0.18 \mathrm{pc})$ at both $\mathrm{P} 1$ and $\mathrm{P} 2$, which are slightly larger than the beam size. P3 is located almost at the edge of the continuum emitting region in the East core.

Molecular gas shows different distributions depending on species. SO is seen at all the positions (P1, P2, P3), while $\mathrm{CS}$ is concentrated in the East core (P2, P3) and the emission is weak at the YSO core. The distribution of CS is more extended compared with other lines and continuum, and the size of the emission region is about $0.8^{\prime \prime}(0.24 \mathrm{pc})$ centered near P2. The CS emission is relatively weak at P1 and a peculiar structure elongated in the north-south direction is seen at the right side of $\mathrm{P} 1$. In contrast to $\mathrm{CS}, \mathrm{H}^{13} \mathrm{CO}^{+}$is concentrated at the YSO core and the source size is comparable to the beam size. $\mathrm{CH}_{3} \mathrm{OH}$ is distributed at both $\mathrm{P} 2$ and $\mathrm{P} 3$, but the emission is slightly stronger at P3. The size of the $\mathrm{CH}_{3} \mathrm{OH}$ emission region around P3 is about $0.4^{\prime \prime}(0.12$ pc), which is almost the same as the beam size. $\mathrm{SO}_{2}$ is seen at both $\mathrm{P} 2$ and $\mathrm{P} 3$ although the $\mathrm{S} / \mathrm{N}$ is not very good. The distribution of $\mathrm{H}_{2} \mathrm{CS}$ seems to be slightly elongated toward the southeast direction from P2. Other lines, including $\mathrm{C}^{33} \mathrm{~S}, \mathrm{SiO}$, and U244.556, are distributed around P2. Note that all of the detected emission distributions are compact as 
Table 2. Line Parameters

\begin{tabular}{|c|c|c|c|c|c|c|c|c|c|}
\hline Molecule & Transition & $\begin{array}{l}E u \\
\text { (K) }\end{array}$ & $\begin{array}{c}\text { Frequency } \\
(\mathrm{GHz})\end{array}$ & $\begin{array}{l}T_{\mathrm{b}} \\
(\mathrm{K})\end{array}$ & $\begin{array}{c}\Delta V \\
\left(\mathrm{~km} \mathrm{~s}^{-1}\right)\end{array}$ & $\begin{array}{c}\int T_{\mathrm{b}} d V \\
\left(\mathrm{~K} \mathrm{~km} \mathrm{~s}^{-1}\right)\end{array}$ & $\begin{array}{c}V_{\mathrm{LSR}} \\
\left(\mathrm{km} \mathrm{s}^{-1}\right)\end{array}$ & $\begin{array}{l}\text { RMS } \\
(\mathrm{K})\end{array}$ & Note \\
\hline \multicolumn{10}{|l|}{ Position 1} \\
\hline CS & $5-4$ & 35 & 244.93556 & $1.27 \pm 0.11$ & 4.0 & $5.45 \pm 1.11$ & 190.2 & 0.19 & $\cdots$ \\
\hline SO & $6_{6}-5_{5}$ & 57 & 258.25583 & $3.64 \pm 0.10$ & 4.0 & $15.61 \pm 0.97$ & 190.6 & 0.18 & $\cdots$ \\
\hline $\mathrm{H}^{13} \mathrm{CO}^{+}$ & $3-2$ & 25 & 260.25533 & $1.75 \pm 0.10$ & 4.4 & $8.24 \pm 1.13$ & 190.3 & 0.19 & $\ldots$ \\
\hline $\mathrm{CH}_{3} \mathrm{OH}$ & $5_{0}-4_{0} \mathrm{~A}^{+}$ & 35 & 241.79143 & $<0.7$ & $\cdots$ & $<1.4$ & $\cdots$ & 0.20 & $\cdots$ \\
\hline $\mathrm{SO}_{2}$ & $5_{2,4}-4_{1,3}$ & 24 & 241.61580 & $<0.4$ & $\cdots$ & $<1.3$ & $\cdots$ & 0.20 & $\cdots$ \\
\hline$C^{33} S$ & $5-4$ & 35 & 242.91361 & $<0.4$ & $\ldots$ & $<1.3$ & $\ldots$ & 0.20 & $\cdots$ \\
\hline${ }^{33} \mathrm{SO}$ & $6_{7}-5_{6}$ & 47 & 259.28403 & $<0.4$ & $\cdots$ & $<1.2$ & $\ldots$ & 0.19 & (1) \\
\hline $\mathrm{c}-\mathrm{C}_{3} \mathrm{H}_{2}$ & $3_{2,1}-2_{1,2}$ & 18 & 244.22215 & $<0.4$ & $\cdots$ & $<1.2$ & $\cdots$ & 0.19 & $\cdots$ \\
\hline \multicolumn{10}{|l|}{ Position 2} \\
\hline $\mathrm{CS}$ & $5-4$ & 35 & 244.93556 & $7.05 \pm 0.12$ & 3.4 & $25.46 \pm 0.91$ & 191.7 & 0.19 & $\cdots$ \\
\hline SO & $6_{6}-5_{5}$ & 57 & 258.25583 & $4.25 \pm 0.11$ & 2.7 & $12.34 \pm 0.77$ & 191.7 & 0.19 & $\ldots$ \\
\hline $\mathrm{H}^{13} \mathrm{CO}^{+}$ & $3-2$ & 25 & 260.25533 & $0.71 \pm 0.12$ & 3.0 & $2.30 \pm 0.96$ & 191.5 & 0.21 & $\ldots$ \\
\hline $\mathrm{CH}_{3} \mathrm{OH}$ & $5_{0}-4_{0} \mathrm{~A}^{+}$ & 35 & 241.79143 & $1.10 \pm 0.12$ & 2.7 & $3.20 \pm 0.75$ & 191.5 & 0.19 & $\cdots$ \\
\hline $\mathrm{CH}_{3} \mathrm{OH}$ & $5_{-1}-4_{-1} \mathrm{E}$ & 40 & 241.76722 & $0.83 \pm 0.11$ & 2.4 & $2.15 \pm 0.62$ & 191.4 & 0.19 & $\cdots$ \\
\hline $\mathrm{CH}_{3} \mathrm{OH}$ & $5_{0}-4_{0} \mathrm{E}$ & 48 & 241.70022 & $0.63 \pm 0.38$ & 1.5 & $0.98 \pm 0.34$ & 191.7 & 0.19 & (2) (3) \\
\hline $\mathrm{CH}_{3} \mathrm{OH}$ & $51_{1}-4_{1} \mathrm{~A}^{-}$ & 50 & 243.91583 & $<0.4$ & $\cdots$ & $<1.2$ & $\cdots$ & 0.19 & $\cdots$ \\
\hline $\mathrm{CH}_{3} \mathrm{OH}$ & $5_{1}-4_{1} E$ & 56 & 241.87907 & $<0.4$ & $\cdots$ & $<1.2$ & $\cdots$ & 0.19 & $\cdots$ \\
\hline $\mathrm{CH}_{3} \mathrm{OH}$ & $5_{ \pm 2}-4_{ \pm 2} \mathrm{E}$ & 61 & 241.90416 & $0.58 \pm 0.38$ & 1.5 & $0.83 \pm 0.32$ & 192.4 & 0.19 & (2) (3) (4) \\
\hline $\mathrm{CH}_{3} \mathrm{OH}$ & $5_{2}-4_{2} \mathrm{~A}^{+}$ & 73 & 241.88771 & $<0.4$ & $\cdots$ & $<1.2$ & $\cdots$ & 0.19 & $\cdots$ \\
\hline $\mathrm{SO}_{2}$ & $5_{2,4}-4_{1,3}$ & 24 & 241.61580 & $0.73 \pm 0.10$ & 3.1 & $2.39 \pm 1.48$ & 192.7 & 0.19 & $\cdots$ \\
\hline$C^{33} \mathrm{~S}$ & $5-4$ & 35 & 242.91361 & $1.14 \pm 0.12$ & 3.7 & $4.50 \pm 1.01$ & 192.1 & 0.19 & $\cdots$ \\
\hline${ }^{33} \mathrm{SO}$ & $6_{7}-5_{6}$ & 47 & 259.28403 & $<0.4$ & $\cdots$ & $<1.3$ & $\ldots$ & 0.21 & (1) \\
\hline $\mathrm{H}_{2} \mathrm{CS}$ & $7_{1,6}-6_{1,5}$ & 60 & 244.04851 & $1.37 \pm 0.11$ & 2.1 & $3.05 \pm 0.64$ & 191.9 & 0.19 & $\cdots$ \\
\hline $\mathrm{SiO}$ & $6-5$ & 44 & 260.51800 & $0.61 \pm 0.12$ & 4.0 & $2.63 \pm 1.12$ & 193.0 & 0.21 & $\ldots$ \\
\hline $\mathrm{H}^{13} \mathrm{CN}$ & $3-2$ & 25 & 259.01181 & $0.66 \pm 0.14$ & 2.1 & $1.47 \pm 0.75$ & 191.1 & 0.21 & $\cdots$ \\
\hline c- $\mathrm{C}_{3} \mathrm{H}_{2}$ & $3_{2,1}-2_{1,2}$ & 18 & 244.22215 & $<0.4$ & $\cdots$ & $<1.2$ & $\ldots$ & 0.19 & $\cdots$ \\
\hline $\mathrm{U} 244.556$ & $\cdots$ & $\cdots$ & 244.5556 & $1.02 \pm 0.11$ & 2.3 & $2.55 \pm 0.68$ & 192.0 & 0.19 & $(5)(6)$ \\
\hline \multicolumn{10}{|l|}{ Position 3} \\
\hline $\mathrm{CS}$ & $5-4$ & 35 & 244.93556 & $6.33 \pm 0.12$ & 2.8 & $18.64 \pm 0.83$ & 192.5 & 0.18 & $\cdots$ \\
\hline SO & $6_{6}-5_{5}$ & 57 & 258.25583 & $4.46 \pm 0.14$ & 2.7 & $12.93 \pm 0.92$ & 192.4 & 0.19 & $\cdots$ \\
\hline $\mathrm{H}^{13} \mathrm{CO}^{+}$ & $3-2$ & 25 & 260.25533 & $0.52 \pm 0.11$ & 2.5 & $1.39 \pm 0.63$ & 192.4 & 0.19 & $\cdots$ \\
\hline $\mathrm{CH}_{3} \mathrm{OH}$ & $5_{0}-4_{0} \mathrm{~A}^{+}$ & 35 & 241.79143 & $1.40 \pm 0.13$ & 2.4 & $3.62 \pm 0.80$ & 192.5 & 0.21 & $\cdots$ \\
\hline $\mathrm{CH}_{3} \mathrm{OH}$ & $5_{-1}-4_{-1} \mathrm{E}$ & 40 & 241.76722 & $0.90 \pm 0.13$ & 2.6 & $2.52 \pm 0.82$ & 192.1 & 0.21 & $\cdots$ \\
\hline $\mathrm{CH}_{3} \mathrm{OH}$ & $5_{0}-4_{0} \mathrm{E}$ & 48 & 241.70022 & $<0.4$ & $\cdots$ & $<1.3$ & $\cdots$ & 0.21 & $\cdots$ \\
\hline $\mathrm{CH}_{3} \mathrm{OH}$ & $5_{1}-4_{1} \mathrm{~A}^{-}$ & 50 & 243.91583 & $<0.4$ & $\cdots$ & $<1.2$ & $\cdots$ & 0.18 & $\cdots$ \\
\hline $\mathrm{CH}_{3} \mathrm{OH}$ & $5_{1}-4_{1} \mathrm{E}$ & 56 & 241.87907 & $<0.4$ & $\cdots$ & $<1.3$ & $\cdots$ & 0.21 & $\cdots$ \\
\hline $\mathrm{CH}_{3} \mathrm{OH}$ & $5_{ \pm 2}-4_{ \pm 2} \mathrm{E}$ & 61 & 241.90416 & $<0.4$ & $\cdots$ & $<1.3$ & $\cdots$ & 0.21 & (4) \\
\hline $\mathrm{CH}_{3} \mathrm{OH}$ & $5_{2}-4_{2} \mathrm{~A}^{+}$ & 73 & 241.88771 & $<0.4$ & $\ldots$ & $<1.3$ & $\ldots$ & 0.21 & $\cdots$ \\
\hline $\mathrm{SO}_{2}$ & $5_{2,4}-4_{1,3}$ & 24 & 241.61580 & $0.97 \pm 0.12$ & 2.0 & $2.10 \pm 0.72$ & 192.2 & 0.21 & $\cdots$ \\
\hline $\mathrm{C}^{33} \mathrm{~S}$ & $5-4$ & 35 & 242.91361 & $<0.4$ & $\cdots$ & $<1.3$ & $\cdots$ & 0.21 & $\cdots$ \\
\hline${ }^{33} \mathrm{SO}$ & $6_{7}-5_{6}$ & 47 & 259.28403 & $<0.4$ & $\cdots$ & $<1.2$ & $\ldots$ & 0.19 & (1) \\
\hline c- $\mathrm{C}_{3} \mathrm{H}_{2}$ & $3_{2,1}-2_{1,2}$ & 18 & 244.22215 & $<0.4$ & $\ldots$ & $<1.2$ & $\ldots$ & 0.18 & $\ldots$ \\
\hline
\end{tabular}

Noте- Uncertainties and upper limits are of the $2 \sigma$ level and do not include systematic errors due to baseline subtraction. Upper limits are estimated assuming $\Delta V=3 \mathrm{~km} \mathrm{~s}^{-1}$. Notes: (1) Two hyperfine components $(\mathrm{F}=15 / 2-13 / 2$ and $\mathrm{F}=17 / 2-15 / 2)$ are blended. (2) Tentative detection. (3) The integrated intensity is calculated by directly integrating the spectrum between $190 \mathrm{~km}$ $\mathrm{s}^{-1}$ and $194 \mathrm{~km} \mathrm{~s}^{-1}$. (4) Two $\mathrm{CH}_{3} \mathrm{OH}$ lines $\left(5_{-2}-4_{-2} \mathrm{E}\right.$ and $\left.5_{2}-4_{2} \mathrm{E}\right)$ are blended. (5) Tentatively attributed to HDS $\left(1_{0,1}-0_{0,0}\right)$ (see Section 4.4). (6) Assuming $V_{\mathrm{LSR}}=192.0 \mathrm{~km} \mathrm{~s}^{-1}$. 

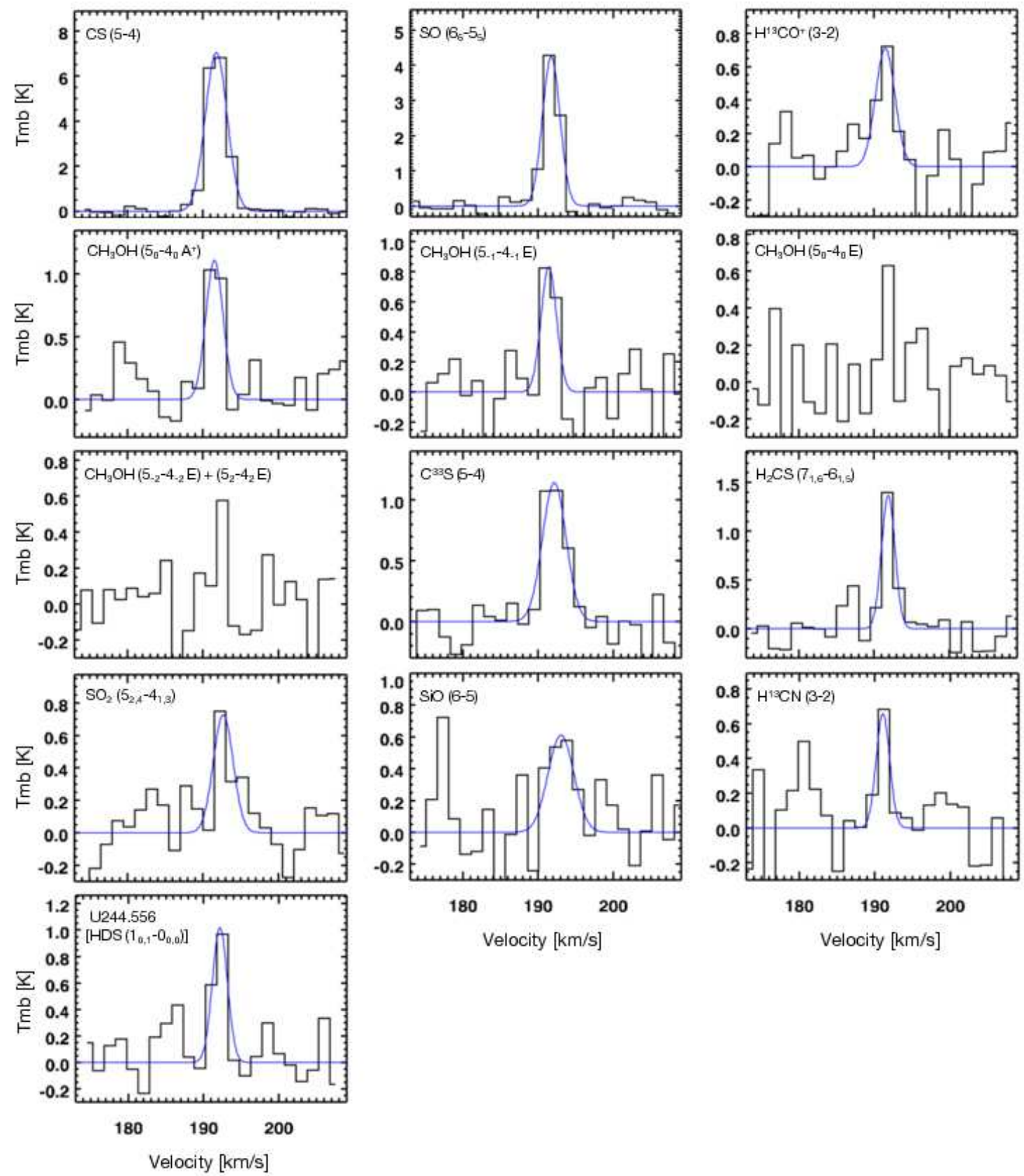

Figure 2. Spectra extracted at Position 2. Emission lines of $\mathrm{CS}, \mathrm{SO}, \mathrm{H}^{13} \mathrm{CO}^{+}, \mathrm{CH}_{3} \mathrm{OH}, \mathrm{C}^{33} \mathrm{~S}, \mathrm{H}_{2} \mathrm{CS}, \mathrm{SO} \mathrm{S}_{2} \mathrm{SiO}, \mathrm{H}^{13} \mathrm{CN}$, and $\mathrm{U} 244.556$ (tentatively attributed to HDS) are shown. The blue lines represent Gaussian profiles fitted to the spectra. For $\mathrm{CH}_{3} \mathrm{OH}\left(5_{0}-4_{0} \mathrm{E}\right)$ and $\left(5_{ \pm 2}-4_{ \pm 2}\right.$ E) lines, integrated intensities are derived by directly integrating the spectrum between $190 \mathrm{~km} \mathrm{~s}^{-1}$ and $194 \mathrm{~km} \mathrm{~s}^{-1}$. 

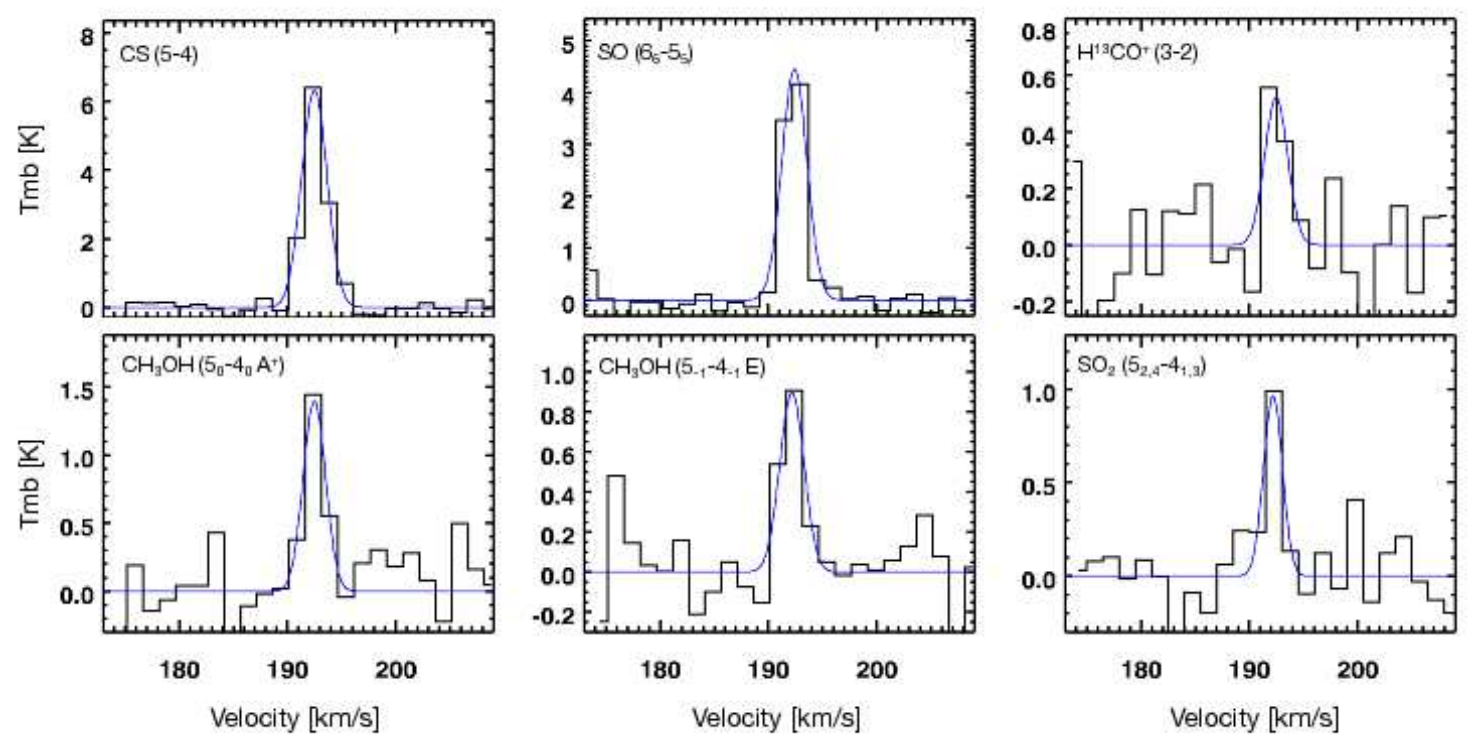

Figure 3. Spectra extracted at Position 3. Emission lines of $\mathrm{CS}, \mathrm{SO}, \mathrm{H}^{13} \mathrm{CO}^{+}, \mathrm{CH}_{3} \mathrm{OH}$, and $\mathrm{SO}_{2}$ are shown. The blue lines represent Gaussian profiles fitted to the spectra.

compared to the maximum recoverable angular scale of the present observations $\left(\sim 3^{\prime \prime}\right)$, and thus the compact emission from the observed region is almost covered with the present array configuration.

A bridge-like structure, which connects the YSO core and the East core, is seen in the continuum, CS, and SO images. Figure 5 shows channel maps for the CS and SO emission lines. A velocity gradient is clearly seen in the figure; the YSO core is relatively blue-shifted while the East core is redshifted, and the bridge region shows an intermediate velocity. The velocity difference among those cores is at most $4 \mathrm{~km}$ $\mathrm{s}^{-1}$. The continuous velocity structure from the YSO core to the bridge, and to the East core, suggests that these regions are dynamically associated.

\subsection{Column densities, gas temperatures, and molecular abundances}

\subsubsection{Rotation diagram analysis for $\mathrm{CH}_{3} \mathrm{OH}$}

We perform the rotation diagram analysis for $\mathrm{CH}_{3} \mathrm{OH}$ because we detect multiple lines with different excitation energies (Figure 6). An optically thin condition and the local thermodynamic equilibrium (LTE) are assumed here. We use the following formulae based on the standard treatment of the rotation diagram analysis (e.g., Sutton et al. 1995; Goldsmith \& Langer 1999):

$$
\frac{N_{u}}{g_{u}}=\frac{3 k \int T_{\mathrm{b}} d V}{8 \pi^{3} v S \mu^{2}}
$$

and

$$
\log \left(\frac{N_{u}}{g_{u}}\right)=-\left(\frac{\log e}{T_{\text {rot }}}\right)\left(\frac{E_{u}}{k}\right)+\log \left(\frac{N}{Q\left(T_{\text {rot }}\right)}\right)
$$

where $N_{u}$ is a column density of molecules in the upper energy level, $g_{u}$ is the degeneracy of the upper level, $k$ is the Boltzmann constant, $\int T_{\mathrm{b}} d V$ is the integrated intensity as estimated from the observations, $v$ is the transition frequency, $S$ is the line strength, $\mu$ is the dipole moment, $T_{\text {rot }}$ is the rotational temperature, $E_{u}$ is the upper state energy, $N$ is a total column density, and $Q\left(T_{\text {rot }}\right)$ is the partition function at $T_{\text {rot }}$. All the spectroscopic parameters required in the analysis are extracted from the CDMS database.

Derived column densities and rotational temperatures are $N=\left(3.0_{-1.9}^{+5.1}\right) \times 10^{14} \mathrm{~cm}^{-2}$ and $T_{\text {rot }}=12.3_{-2.9}^{+5.3} \mathrm{~K}$ for Position 2 , and $N=\left(3.2_{-1.9}^{+4.5}\right) \times 10^{14} \mathrm{~cm}^{-2}$ and $T_{\text {rot }}=13.1_{-2.8}^{+4.9}$ $\mathrm{K}$ for Position 3 ( $2 \sigma$ uncertainties). Note that the measured intensity of the $\mathrm{CH}_{3} \mathrm{OH}$ line at $241.90416 \mathrm{GHz}$ is divided by two, because it is a blend of two components $\left(5_{-2}-4_{-2} \mathrm{E}\right.$ and $5_{2}-4_{2}$ E) with similar spectroscopic properties.

In the above analysis, the data points for two marginal $\mathrm{CH}_{3} \mathrm{OH}$ detections $\left(5_{0}-4_{0} \mathrm{E}\right.$ and $5_{ \pm 2}-4_{ \pm 2} \mathrm{E}, \mathrm{Eu}=48 \mathrm{~K}$ and $61 \mathrm{~K})$ are included in the straight-line fit for $\mathrm{P} 2$. For P3, two upper limit points for $\mathrm{CH}_{3} \mathrm{OH}\left(5_{0}-4_{0} \mathrm{E}\right)$ and $\left(5_{1}-4_{1} \mathrm{~A}^{-}\right)(E u$ $=50 \mathrm{~K})$ are included in the fit. If we neglect these marginal detections or upper limits in the fit, we obtain higher temperatures and slightly lower column densities; for P2, $T_{\text {rot }}=15.6$ $\mathrm{K}$ and $N=2.5 \times 10^{14} \mathrm{~cm}^{-2}$, and for P3, $T_{\text {rot }}=17.3 \mathrm{~K}$ and $N=2.8 \times 10^{14} \mathrm{~cm}^{-2}$. However, these fits are incompatible with upper limits, and the fitted line deviates upward from the points. Thus, we take account of the above two detections in P2 and the two upper limit data in P3 to better fit the observation data. 


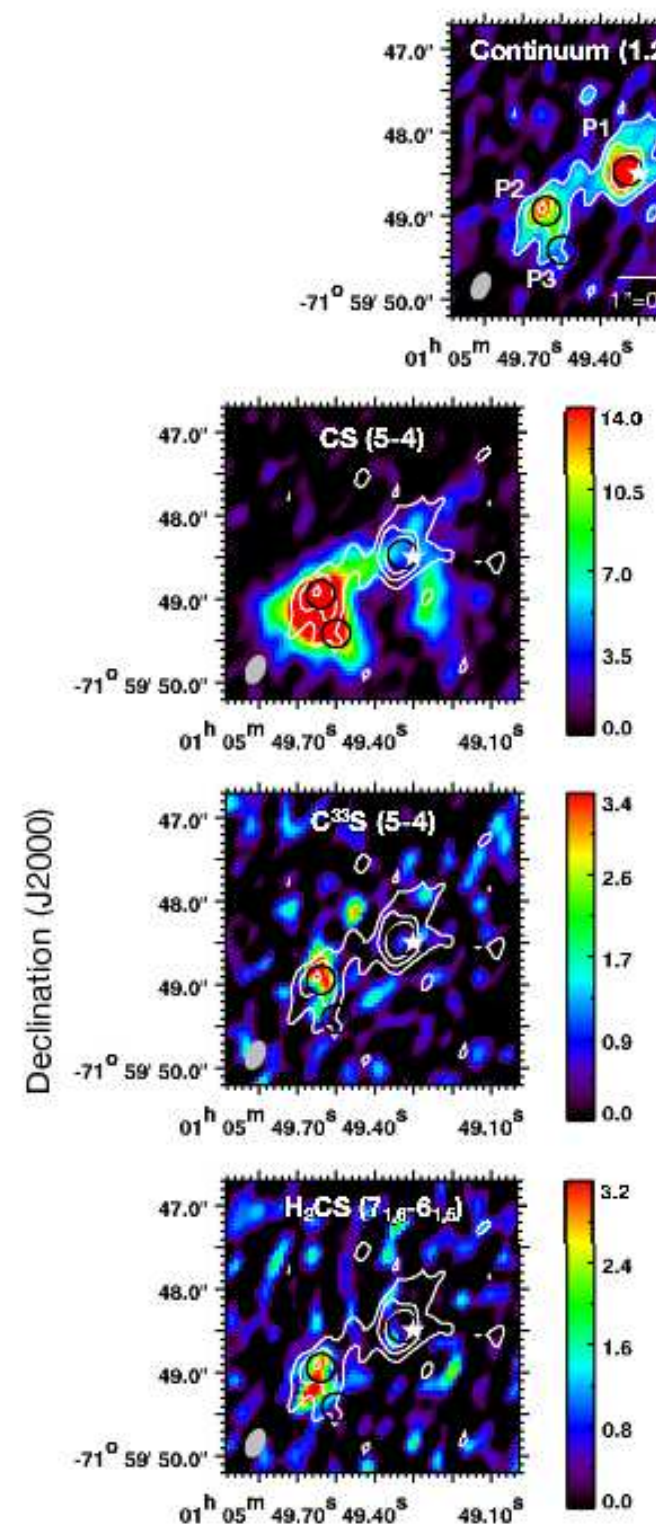

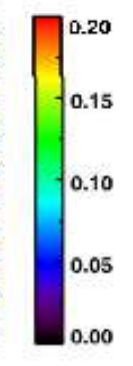

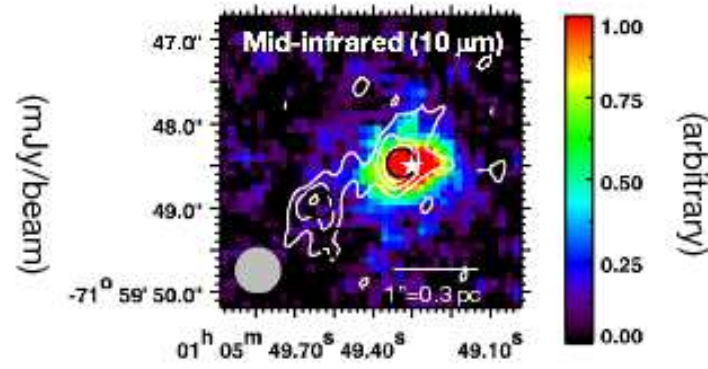

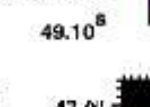

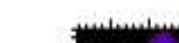
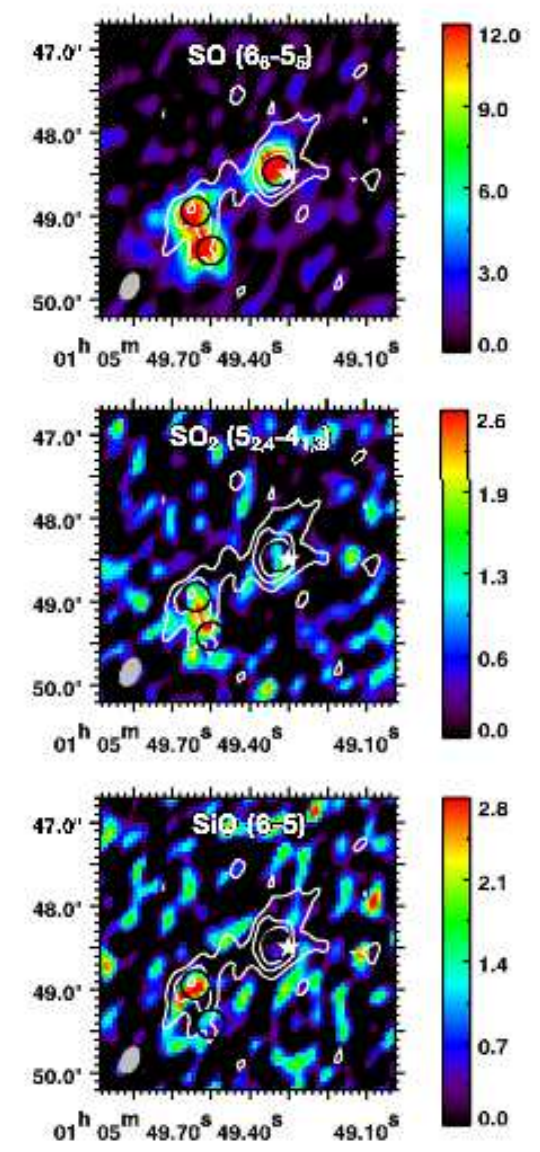

Right Ascension (J2000)
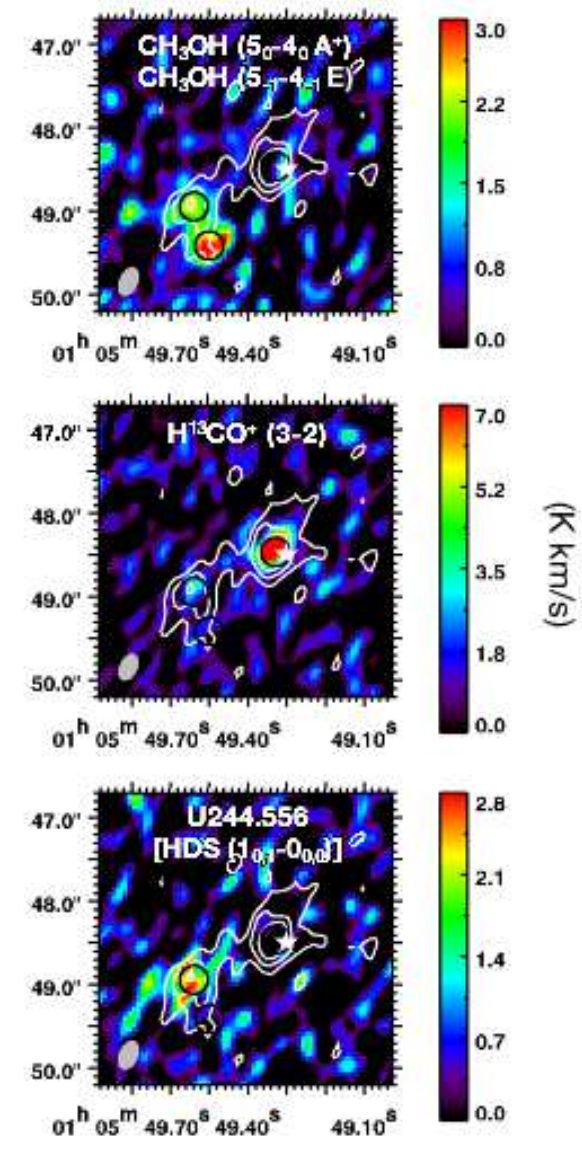

Figure 4. Flux distributions of the ALMA $1.2 \mathrm{~mm}$ continuum (top left) and the Gemini/T-ReCS mid-infrared $10 \mu \mathrm{m}$ emission (top right), and integrated intensity distributions of $\mathrm{CS}, \mathrm{SO}, \mathrm{CH}_{3} \mathrm{OH}, \mathrm{C}^{33} \mathrm{~S}, \mathrm{SO}_{2}, \mathrm{H}^{13} \mathrm{CO}^{+}, \mathrm{H}_{2} \mathrm{CS}$, $\mathrm{SiO}$, and $\mathrm{U} 244.556$ (tentatively attributed to $\mathrm{HDS}$ ). Contours represent the $1.2 \mathrm{~mm}$ continuum distribution and the contour levels are $3 \sigma, 6 \sigma$, and $10 \sigma$ of the rms noise. The synthesized beam size $(0.11 \mathrm{pc} \times$ $0.07 \mathrm{pc}$ at the SMC) is shown by the gray filled ellipse in each panel except for the $10 \mu \mathrm{m}$ data, whose circle represents an FWHM of a standard star's point spread function. The spectra discussed in the text are extracted from the three positions (P1, P2, and P3) indicated by black open circles in each panel. The white filled star represents the position of an embedded high-mass YSO identified by infrared observations. North is up, and east is to the left. 


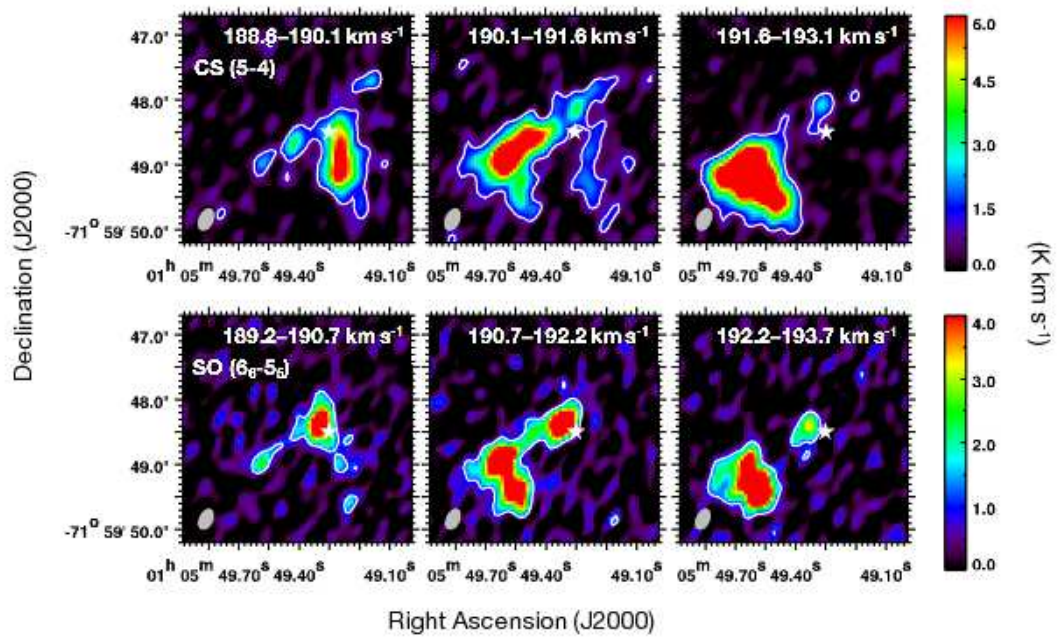

Figure 5. Channel maps for CS (up) and SO (bottom) at three velocity ranges (CS: 188.6-190.1, 190.1-191.6, 191.6-193.1 km s ${ }^{-1}$; SO: $189.2-190.7,190.7-192.2,192.2-193.7 \mathrm{~km} \mathrm{~s}^{-1}$ ). The plotted symbols are the same as in Figure 4 . The contours represent the regions above $4 \sigma$ of the rms noise, and the filled star is the position of an embedded high-mass YSO. A velocity gradient from the YSO core to the East core is seen in the figure. 

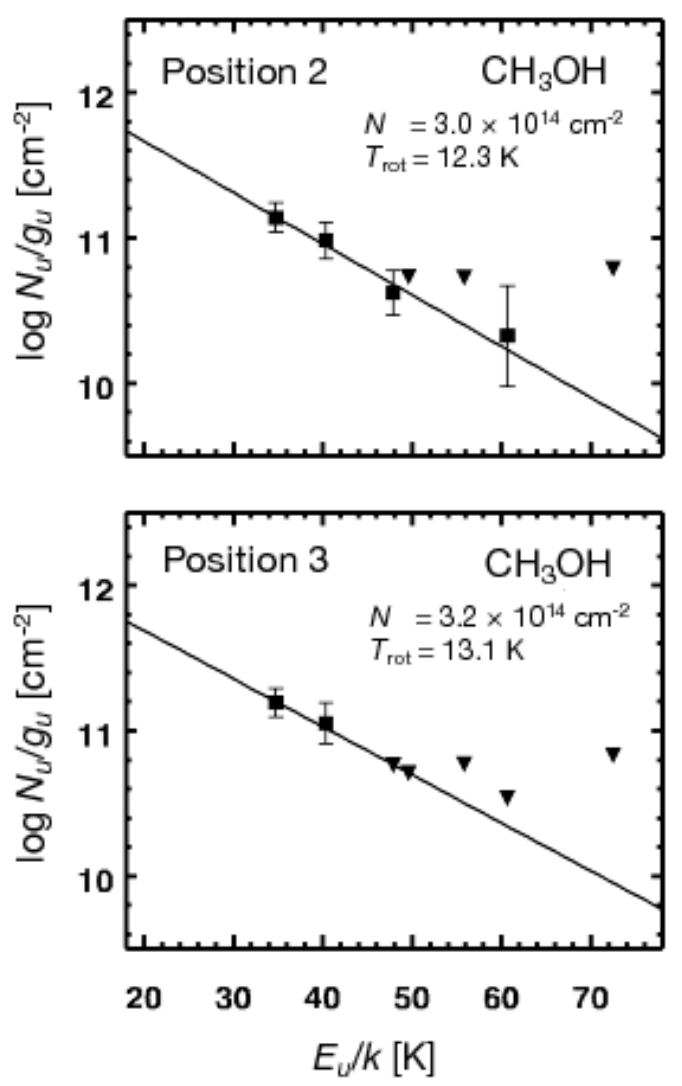

Figure 6. Rotation diagrams for $\mathrm{CH}_{3} \mathrm{OH}$ lines at Position 2 (top) and 3 (bottom). Upper-limit points are shown by the downwardpointing triangles. The solid lines represent the fitted straight line. Derived column densities and rotation temperatures are indicated in each panel. Note that A- and E-state $\mathrm{CH}_{3} \mathrm{OH}$ molecules are fitted simultaneously. See Section 3.3.1 for details.

Note that the presence of warm gas components $(\gtrsim 50 \mathrm{~K})$ within the core can not be ruled out from the limited frequency coverage of the present observations. Future observations in higher frequency regions are thus desirable.

\subsubsection{Column density of $\mathrm{H}_{2}$, dust extinction, and mass}

A column density of molecular hydrogen $\left(N_{\mathrm{H}_{2}}\right)$ is estimated from the dust continuum data obtained in this work. The measured continuum brightnesses are $0.30 \mathrm{mJy} / \mathrm{beam}$ for P1, $0.16 \mathrm{mJy} /$ beam for $\mathrm{P} 2$, and $0.06 \mathrm{mJy} /$ beam for $\mathrm{P} 3$. The following equation is used to calculate $N_{\mathrm{H}_{2}}$ based on the standard treatment of optically thin dust emission (e.g., Shimonishi et al. 2016b):

$$
N_{\mathrm{H}_{2}}=\frac{F_{v} / \Omega}{2 \kappa_{v} B_{v}\left(T_{d}\right) Z \mu m_{\mathrm{H}}},
$$

where $F_{v} / \Omega$ is the continuum flux density per beam solid angle as estimated from the observations, $\kappa_{v}$ is the mass absorption coefficient of dust grains coated by thin ice mantles as taken from Ossenkopf \& Henning (1994, here we use 1.1 $\left.\mathrm{cm}^{2} \mathrm{~g}^{-1}\right), T_{d}$ is the dust temperature, $B_{v}\left(T_{d}\right)$ is the Planck function, $Z$ is the dust-to-gas mass ratio, $\mu$ is the mean atomic mass per hydrogen (1.41, according to Cox 2000), and $m_{\mathrm{H}}$ is the hydrogen mass. We assume that the dust-to-gas mass ratio in the SMC is lower than the typical Galactic value of 0.008 by a factor of five according to the metallicity. This assumption would be valid in a high density regime although dust-to-gas mass ratios in SMC diffuse clouds are higher than the value adopted here (e.g., Roman-Duval et al. 2017).

The dust temperature at $\mathrm{P} 2$ and $\mathrm{P} 3$ is assumed to be $12 \mathrm{~K}$ and $13 \mathrm{~K}$ according to the rotation temperatures of $\mathrm{CH}_{3} \mathrm{OH}$ under the assumption of the LTE. In sub-thermal conditions, however, the kinetic temperature of gas is often higher than excitation temperatures. If we assume a slightly elevated dust temperature, i.e. $T_{d}=20 \mathrm{~K}$, the derived $\mathrm{H}_{2}$ column densities decrease by a factor of two, and thus fractional molecular abundances are doubled. We should keep in mind that derived molecular abundances entail the systematic uncertainty depending on the assumption of dust temperatures. The dust temperature at $\mathrm{P} 1$ is unknown. We thus vary the dust temperature from $10 \mathrm{~K}$ to $20 \mathrm{~K}$ to derive the range of possible $\mathrm{H}_{2}$ column densities. Assumption of higher dust temperatures $\left(T_{d}>23 \mathrm{~K}\right)$ for P1 leads to a low $A_{V}$ value $\left(A_{V}<8 \mathrm{mag}\right)$, which is unrealistic as extinction towards a deeply embedded high-mass YSO, and also inconsistent with the previous $A_{V}$ estimate described below.

The derived $\mathrm{H}_{2}$ column densities are shown in Table 3 and used to calculate fractional abundances of other molecular species. The total gas mass contained in each core is estimated to be about $80 \mathrm{M}_{\odot}$ for the YSO core and about $50 \mathrm{M}_{\odot}$ for the East core (assuming $T_{d}=10 \mathrm{~K}$ for both cores).

We also estimate visual extinction $\left(A_{V}\right)$ using an $N_{\mathrm{H}_{2}} / A_{V}$ conversion factor. Martin et al. (1989) reported $N_{\mathrm{H}} / E(B-V)$ $=4.6 \times 10^{22} \mathrm{~cm}^{-2} \mathrm{mag}^{-1}$ for the SMC. Using $A_{V} / E(B-V)$ $\sim 4$ for dense clouds (Whittet et al. 2001), we obtain $N_{\mathrm{H}_{2}} / A_{V}$ $=5.8 \times 10^{21} \mathrm{~cm}^{-2} \mathrm{mag}^{-1}$, where all the hydrogen atoms are assumed to be in $\mathrm{H}_{2}$. A similar ratio is obtained when the Galactic canonical $N_{\mathrm{H}} / A_{V}$ value $\left(=1.9 \times 10^{21} \mathrm{~cm}^{-2} \mathrm{mag}^{-1}\right)$ is scaled by the metallicity of the SMC. Using the above $N_{\mathrm{H}_{2}} / A_{V}$ ratio, we estimate $A_{V} \sim 10-30$ mag at P1, $\sim 10$ mag at P2, and $\sim 4$ mag at P3. Ward et al. (2017) estimate $A_{V}=16$ \pm 8 mag for the YSO at P1 using the ratio of near-infrared $\mathrm{H}_{2}$ emission lines; the value agrees with our $A_{V}$ estimate that is based on the submillimeter continuum. Note that these are rough estimates since the $N_{\mathrm{H}_{2}} / A_{V}$ ratio is highly uncertain for dense clouds in the SMC.

\subsubsection{Column densities of other molecules}

Column densities of molecular species other than $\mathrm{CH}_{3} \mathrm{OH}$ and $\mathrm{H}_{2}$ are estimated by solving Equation 2 for $N$. As well as the dust continuum analysis described in Section 3.3.2, the 
gas temperature at P2 and P3 is assumed to be $12 \mathrm{~K}$ and 13 $\mathrm{K}$, while the gas temperature at $\mathrm{P} 1$ is varied between $10 \mathrm{~K}$ and $20 \mathrm{~K}$.

The CS(5-4) and $\mathrm{SO}\left(6_{6}-5_{5}\right)$ lines are often optically thick in star-forming regions. For $\mathrm{CS}$ at $\mathrm{P} 1$ and $\mathrm{P} 3$, we use the $\mathrm{C}^{32} \mathrm{~S}$ data to derive a lower limit and the $C^{33} S$ non-detection data for an upper limit to estimate a possible range of column densities. At P2, the CS column density is derived only from the $\mathrm{C}^{33} \mathrm{~S}$ line. Similarly, for SO, we use the ${ }^{32} \mathrm{SO}$ data to derive a lower limit and the ${ }^{33} \mathrm{SO}$ non-detection data for an upper limit to estimate a possible range of column densities at all positions. We here assume ${ }^{32} \mathrm{~S} /{ }^{33} \mathrm{~S}=40$, based on the ${ }^{33} \mathrm{SO}_{2}$ observations towards a hot core in the LMC (Shimonishi et al. 2016b). Column densities of $\mathrm{HCO}^{+}$and $\mathrm{HCN}$ are estimated from $\mathrm{H}^{13} \mathrm{CO}^{+}$and $\mathrm{H}^{13} \mathrm{CN}$ data, respectively. We here assume ${ }^{12} \mathrm{C} /{ }^{13} \mathrm{C}=50$ according to $\mathrm{HCO}^{+} / \mathrm{H}^{13} \mathrm{CO}^{+}$observations of the $\mathrm{N} 27$ region in the SMC (Heikkilä et al. 1999).

Estimated column densities and fractional abundances at each position are summarized in Table 3.

\section{DISCUSSION}

\subsection{Physical properties of two dense cores}

The present ALMA observations resolved a single infrared source into two molecular cloud cores (the YSO core and the East core). They are physically associated but show different chemical properties. We first discuss physical properties of these cores in the following section.

\subsubsection{The YSO core}

The molecular cloud core including Position 1 is associated with an embedded high-mass YSO that is bright in infrared and radio continuum. A spectral energy distribution (SED) of the YSO is shown in Figure 7 (data are collected from available databases and literatures, including Skrutskie et al. 2006; Meixner et al. 2006, 2013; Kemper et al. 2010). The photometric point at $1200 \mu \mathrm{m}$ is estimated from the present ALMA data. The $2-5 \mu \mathrm{m}$ spectrum is based on our $A K A R I$ data, and the five narrow-band photometric points around $10 \mu \mathrm{m}$ are based on our Gemini/TReCS data (see Section 2.4 for details of these data). The YSO's SED peaks in the far-infrared region $(\sim 50-70 \mu \mathrm{m})$, suggesting that the source is at an early evolutionary stage. The bolometric luminosity of the YSO is estimated to be 2 $\times 10^{4} \mathrm{~L}_{\odot}$ by integrating the SED from $1 \mu \mathrm{m}$ to $1200 \mu \mathrm{m}$. Oliveira et al. (2013) reported a luminosity of $2.3 \times 10^{4} \mathrm{~L}_{\odot}$ for the YSO, with which our estimate is consistent. The high luminosity is consistent with the presence of a high-mass YSO.

Absorption bands due to the $\mathrm{H}_{2} \mathrm{O}$ ice, the $\mathrm{CO}_{2}$ ice, and the silicate dust are seen in the $2-20 \mu \mathrm{m}$ spectrum of the YSO (Fig. 7), as previously reported in Oliveira et al. (2011, 2013). The beam size of the AKARI data, which shows the

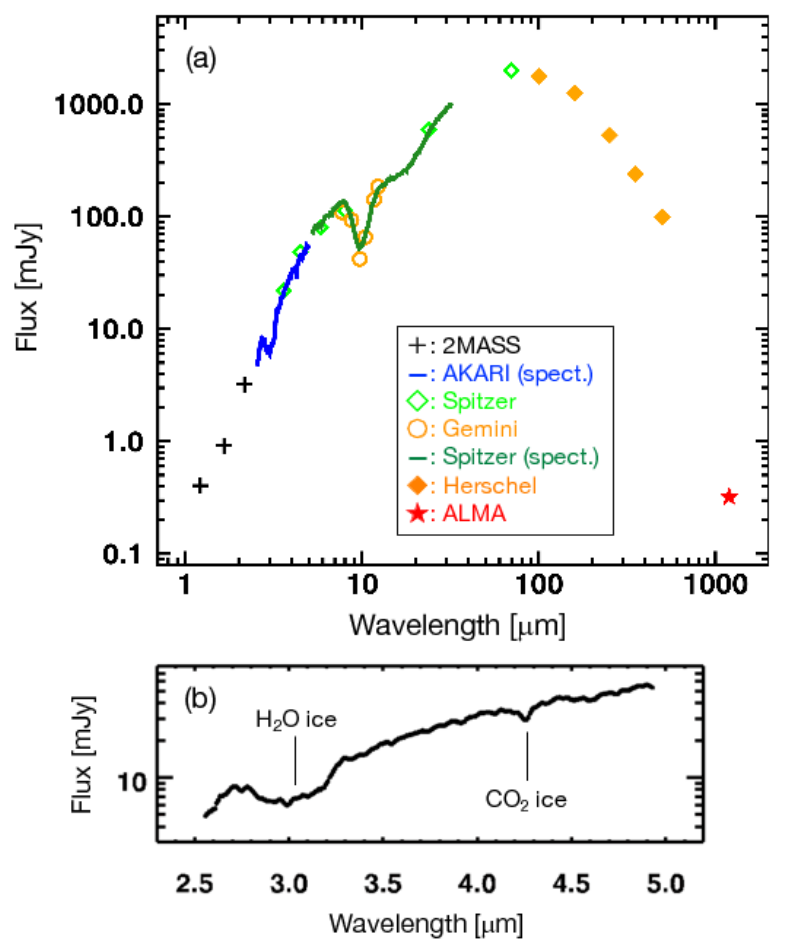

Figure 7. (a): The SED of a high-mass YSO located at Position 1. The plotted data are based on $2 \mathrm{MASS} \mathrm{JHK}_{\mathrm{s}}$ photometry (pluses, black), AKARI/IRC spectroscopy (solid line, blue), Spitzer/IRAC and MIPS photometry (open diamonds, light green), Gemini/T-ReCS narrow-band photometry (open circles, orange), Spitzer/MIPS spectroscopy (solid line, green), Herschel/PACS and SPIRE photometry (filled diamonds, orange), and ALMA $1200 \mu \mathrm{m}$ continuum measurement obtained in this work (filled star,red). (b): Zoom-in of the $2-5 \mu \mathrm{m}$ spectrum obtained by AKARI. The positions of the $3.05 \mu \mathrm{m} \mathrm{H}_{2} \mathrm{O}$ ice band and the $4.27 \mu \mathrm{m} \mathrm{CO}_{2}$ ice band are labeled. See Section 4 for more details.

ice absorption bands, is much larger than those of ALMA and Gemini data. However, because ices are observed with absorption spectroscopy, the absence of an infrared continuum source at P2 and P3 as shown in the infrared image indicates that the observed ice absorption bands originate mainly from the infrared point source associated with P1. Further analysis on ice absorption bands is described in Section 4.2. Previous studies detected compact emission due to hydrogen recombination lines and shocked $\mathrm{H}_{2}$ lines associated with the YSO (Oliveira et al. 2013; Ward et al. 2017). On the other hand, emission features due to fine-structure lines from ionized metals and polycyclic aromatic hydrocarbon (PAH) bands are absent or weak in the infrared spectrum of the YSO. The above spectral characteristics suggest that the YSO is still embedded in cold dust and ices, and a prominent $\mathrm{H}$ II region is not formed yet.

A number density of molecular hydrogen at $\mathrm{P} 1$ is estimated to be $n_{\mathrm{H}_{2}} \sim 3-8 \times 10^{5} \mathrm{~cm}^{-3}$ (assuming a source diameter of 
Table 3. Column densities and fractional abundances

\begin{tabular}{|c|c|c|c|c|c|c|}
\hline \multirow[b]{2}{*}{ Molecule } & \multicolumn{2}{|c|}{ Position $1^{\mathrm{a}}$} & \multicolumn{2}{|c|}{ Position $2^{\mathrm{b}}$} & \multicolumn{2}{|c|}{ Position $3^{b}$} \\
\hline & $\begin{array}{c}N \\
\left(\mathrm{~cm}^{-2}\right)\end{array}$ & $N / N_{\mathrm{H}_{2}}$ & $\begin{array}{c}N \\
\left(\mathrm{~cm}^{-2}\right)\end{array}$ & $N / N_{\mathrm{H}_{2}}$ & $\begin{array}{c}N \\
\left(\mathrm{~cm}^{-2}\right)\end{array}$ & $N / N_{\mathrm{H}_{2}}$ \\
\hline $\mathrm{H}_{2}{ }^{\mathrm{c}}$ & $(0.6-1.6) \times 10^{23}$ & $\cdots$ & $6.3 \times 10^{22}$ & $\cdots$ & $2.1 \times 10^{22}$ & $\cdots$ \\
\hline $\mathrm{CH}_{3} \mathrm{OH}^{\mathrm{d}}$ & $(<1.1-1.8) \times 10^{14}$ & $<2 \times 10^{-9}$ & $\left(3.0_{-1.9}^{+5.1}\right) \times 10^{14}$ & $\left(4.7_{-3.0}^{+8.0}\right) \times 10^{-9}$ & $\left(3.2_{-1.9}^{+4.5}\right) \times 10^{14}$ & $\left(1.5_{-0.9}^{+2.2}\right) \times 10^{-8}$ \\
\hline $\mathrm{CS}^{\mathrm{e}}$ & $(0.2-5.4) \times 10^{14}$ & $(1.8 \pm 1.5) \times 10^{-9}$ & $(1.3 \pm 0.3) \times 10^{15}$ & $(2.0 \pm 0.4) \times 10^{-8}$ & $(1.1-3.3) \times 10^{14}$ & $(1.0 \pm 0.5) \times 10^{-8}$ \\
\hline $\mathrm{SO}^{\mathrm{f}}$ & $(0.5-6.4) \times 10^{15}$ & $(2.4 \pm 1.6) \times 10^{-8}$ & $(1.4-4.0) \times 10^{15}$ & $(4.3 \pm 2.1) \times 10^{-8}$ & $(1.1-3.0) \times 10^{15}$ & $(9.5 \pm 4.5) \times 10^{-8}$ \\
\hline $\mathrm{SO}_{2}$ & $(<5.2-6.0) \times 10^{13}$ & $<9 \times 10^{-10}$ & $(9.9 \pm 6.1) \times 10^{13}$ & $(1.6 \pm 1.0) \times 10^{-9}$ & $(8.4 \pm 2.9) \times 10^{13}$ & $(4.0 \pm 1.4) \times 10^{-9}$ \\
\hline $\mathrm{H}_{2} \mathrm{CS}$ & $(<0.3-1.8) \times 10^{14}$ & $<1 \times 10^{-9}$ & $(2.4 \pm 0.5) \times 10^{14}$ & $(3.8 \pm 0.8) \times 10^{-9}$ & $<6.9 \times 10^{13}$ & $<3 \times 10^{-9}$ \\
\hline $\mathrm{HCN}^{\mathrm{g}}$ & $(<5.1-9.1) \times 10^{13}$ & $<9 \times 10^{-10}$ & $(9.6 \pm 4.4) \times 10^{13}$ & $(1.4 \pm 0.7) \times 10^{-9}$ & $<6.5 \times 10^{13}$ & $<3 \times 10^{-9}$ \\
\hline $\mathrm{HCO}^{+\mathrm{g}}$ & $(1.7-4.1) \times 10^{14}$ & $(2.9 \pm 1.0) \times 10^{-9}$ & $(7.9 \pm 3.3) \times 10^{13}$ & $(1.3 \pm 0.5) \times 10^{-9}$ & $(4.4 \pm 2.0) \times 10^{13}$ & $(2.1 \pm 1.0) \times 10^{-9}$ \\
\hline c- $-\mathrm{C}_{3} \mathrm{H}_{2}$ & $(<5.5-6.3) \times 10^{13}$ & $<1 \times 10^{-9}$ & $<5.3 \times 10^{13}$ & $<9 \times 10^{-10}$ & $<5.1 \times 10^{13}$ & $<2 \times 10^{-9}$ \\
\hline $\mathrm{SiO}$ & $(<0.2-1.1) \times 10^{13}$ & $<7 \times 10^{-11}$ & $(1.3 \pm 0.6) \times 10^{13}$ & $(2.1 \pm 0.9) \times 10^{-10}$ & $<7.9 \times 10^{12}$ & $<4 \times 10^{-10}$ \\
\hline
\end{tabular}

Note- Uncertainties and upper limits are of the $2 \sigma$ level and do not include systematic errors due to adopted spectroscopic constants. ${ }^{a} T_{\text {rot }}$ varied between $10 \mathrm{~K}$ and $20 \mathrm{~K} .{ }^{b} T_{\text {rot }}$ assumed to be $12 \mathrm{~K}$ for P2 and $13 \mathrm{~K}$ for P3 based on the rotation analysis of $\mathrm{CH}_{3} \mathrm{OH}$ lines. ${ }^{c}$ Estimated from dust continuum. ${ }^{d}$ Estimated based on the rotation analysis. ${ }^{e}$ The lower limit and the upper limit are estimated from the $\mathrm{CS}$ and $\mathrm{C}^{33} \mathrm{~S}$ data (assuming ${ }^{32} \mathrm{~S} /{ }^{33} \mathrm{~S}=40$ ). For Position 2 , only the $\mathrm{C}^{33} \mathrm{~S}$ data are used. ${ }^{f}$ The lower limit and the upper limit are estimated from the SO and ${ }^{33} \mathrm{SO}$ data using the above isotope ratio. ${ }^{g}$ Estimated from the isotopologue assuming ${ }^{12} \mathrm{C} /{ }^{13} \mathrm{C}=50$. See Section 3.3 for details.

$0.1 \mathrm{pc}$ and a spherical distribution of gas) and the $A_{V}$ in the line of sight is $\sim 10-30 \mathrm{mag}$. This suggests that the YSO core contains dense molecular gas that is well shielded from the external radiation field. Given the embedded nature of the source and the absence of a prominent $\mathrm{H}$ II region, the radiation from the central protostar would only have a limited effect on the bulk of molecular gas within the core.

\subsubsection{The East core}

The molecular cloud core including Position 2 and 3 is not associated with an infrared source. The gas density (assuming the source diameter of $0.1 \mathrm{pc}$ and the spherical distribution) and the dust extinction are estimated to be $n_{\mathrm{H}_{2}}=3 \times$ $10^{5} \mathrm{~cm}^{-3}$ and $A_{V} \sim 10 \mathrm{mag}$ at P2, while $n_{\mathrm{H}_{2}}=1 \times 10^{5} \mathrm{~cm}^{-3}$ and $A_{V} \sim 4$ mag at P3. The temperature of molecular gas is $\sim 10 \mathrm{~K}$ as estimated from $\mathrm{CH}_{3} \mathrm{OH}$ lines (see Section 3.3.1). These properties suggest that the East core contains cold and dense molecular gas.

The lack of infrared sources suggests that a high-mass YSO is not present in the East core except for extremely young and cold ones that are elusive even in infrared. However, the possible presence of low-mass YSOs in the East core cannot be ruled out from the current infrared data because of the sensitivity limit. A single low-mass YSO would be too faint to be detected by the present submillimeter observations since the size of a protostellar core is usually small ( $\lesssim 0.01$ pc, e.g., van Dishoeck et al. 1995), but an unresolved cluster of low-mass YSOs may produce a detectable amount of molecular line emission. A massive starless cloud such as an infrared dark cloud is also a possible candidate for the East core.

The low temperature of $\mathrm{CH}_{3} \mathrm{OH}$ gas, as well as the lack of a bright infrared source in the East core, suggests that the presence of a prominent hot core would be less likely. Cold $\mathrm{CH}_{3} \mathrm{OH}$ gas $(\sim 5 \mathrm{~K})$ has been detected in Galactic low-mass starless cores, but the observed line width is typically $0.1-0.5$ $\mathrm{km} \mathrm{s}^{-1}$, which is much narrower than the line width measured for the East core (Tafalla et al. 2006; Soma et al. 2015).

The above discussion suggests that the East core would harbor (i) a massive starless core, or (ii) an embedded highmass YSO(s) before the emergence of infrared emission, or (iii) a cluster of low-mass embedded YSOs.

\subsection{Chemical differentiation}

Chemical compositions of the YSO core and the East core, which are separated by about $0.3 \mathrm{pc}$, show different characteristics. Figure 8 compares molecular abundances at P1, P2, and $\mathrm{P} 3$. The $\mathrm{P} 1-\mathrm{P} 2$ comparison shows that only $\mathrm{HCO}^{+}$is 
more abundant at $\mathrm{P} 1$, while $\mathrm{CS}, \mathrm{H}_{2} \mathrm{CS}$, and $\mathrm{SiO}$ are significantly underabundant at P1. On the other hand, the P2-P3 comparison shows generally similar molecular abundances between $\mathrm{P} 2$ and $\mathrm{P} 3$. Note that $\mathrm{CH}_{3} \mathrm{OH}$ and $\mathrm{SO}_{2}$ are slightly overabundant at P3 compared to P2. The correlation coefficient for abundances of molecular species that are detected in both $\mathrm{P} 2$ and $\mathrm{P} 3\left(\mathrm{CH}_{3} \mathrm{OH}, \mathrm{CS}, \mathrm{SO}, \mathrm{SO}_{2}\right.$, and $\left.\mathrm{HCO}^{+}\right)$is 0.89. The good correlation suggests a similar molecular abundances within the East core. These results imply a chemical differentiation between the YSO core and the East core.

One possible interpretation of this chemical differentiation is the different degree of the adsorption of gas-phase species onto grain surfaces. As shown in Figure 7(b), absorption bands due to the $\mathrm{H}_{2} \mathrm{O}$ and $\mathrm{CO}_{2}$ ices are detected in the line of sight toward the YSO located at P1. The estimated high $A_{V}$ toward P1 (10-30 mag) is actually sufficient for the formation of ice mantles. Using the AKARI spectrum shown in the figure, ice column densities are estimated to be $N\left(\mathrm{H}_{2} \mathrm{O}\right.$ ice $)=(17 \pm 5) \times 10^{17} \mathrm{~cm}^{-2}$ and $N\left(\mathrm{CO}_{2}\right.$ ice $)=$ $(1.1 \pm 0.2) \times 10^{17} \mathrm{~cm}^{-2}$ for the absorption bands at $3.05 \mu \mathrm{m}$ and $4.27 \mu \mathrm{m}$ (derivation is based on the method presented in Shimonishi et al. 2016a). For the $\mathrm{CH}_{3} \mathrm{OH}$ and $\mathrm{CO}$ ices, upper limits of $N\left(\mathrm{CH}_{3} \mathrm{OH}\right.$ ice $) \leq 4 \times 10^{17} \mathrm{~cm}^{-2}$ and $N(\mathrm{CO}$ ice $)$ $\leq 2 \times 10^{17} \mathrm{~cm}^{-2}(2 \sigma)$ are estimated for the absorption bands at $3.53 \mu \mathrm{m}$ and $4.67 \mu \mathrm{m}$, respectively. Oliveira et al. (2011, 2013) have estimated ice column densities of the YSO to be $N\left(\mathrm{H}_{2} \mathrm{O}\right.$ ice $)=(16.6 \pm 0.7) \times 10^{17} \mathrm{~cm}^{-2}, N\left(\mathrm{CO}_{2}\right.$ ice $)=(1.0 \pm$ $0.2) \times 10^{17} \mathrm{~cm}^{-2}$, and $N(\mathrm{CO}$ ice $) \leq 0.45 \times 10^{17} \mathrm{~cm}^{-2}$ using ground-based L-/M-band spectra and a Spitzer mid-infrared spectrum. The present results of the AKARI spectroscopy agree with their measurements.

The total fractional abundance of elemental oxygen (w.r.t. $\mathrm{H}$ ) in solid $\mathrm{H}_{2} \mathrm{O}$ and $\mathrm{CO}_{2}$ at $\mathrm{P} 1$ is estimated to be $\sim 1 \times 10^{-5}$, where we use the $\mathrm{H}_{2}$ column density shown in Table 3. Similarly, that for elemental carbon in ice mantles is estimated to be $\sim 6 \times 10^{-7}$. Elemental abundances of gas-phase oxygen and carbon in the SMC, after considering the depletion into dust grain material, are expected to be $3.7 \times 10^{-5}$ for oxygen and $1.5 \times 10^{-5}$ for carbon, according to the the SMC lowmetal model of Acharyya \& Herbst (2016). Although the gas-phase counterparts of $\mathrm{H}_{2} \mathrm{O}$ and $\mathrm{CO}_{2}$ are not observed in this work, the above discussion suggests that a non-negligible fraction of heavy elements, especially oxygen, are frozen in ice mantles at P1.

Shock chemistry is another possible cause of the chemical differentiation between the YSO core and the East core. The detection of shock tracers such as $\mathrm{SO}_{2}$ and $\mathrm{SiO}$ at P2/P3 implies a possible contribution of shock chemistry on the chemical compositions. Ward et al. (2017) detected collisionallyexcited near-infrared $\mathrm{H}_{2}$ emission lines in IRAS 01042-7215, which suggests the presence of shocked gas in this region.
The effect of shocks on the chemical compositions of the East core will be further discussed in Section 4.3.2.

Finally, we note that future high-sensitivity observations for ices in the East core will be important to further investigate the chemical differentiation in the observed region, since the estimated $A_{V}$ at P2 and P3 ( 4-10 mag) is still plausible for the ice formation.

\subsection{Organic molecules in a low metallicity environment}

The detection of $\mathrm{CH}_{3} \mathrm{OH}$ in the SMC has a strong impact on our understanding of the formation of organic molecules in metal-poor environments. We here discuss abundances and possible origins of cold $\mathrm{CH}_{3} \mathrm{OH}$ gas in the East core.

\subsubsection{Abundance of $\mathrm{CH}_{3} \mathrm{OH}$}

The fractional abundance of $\mathrm{CH}_{3} \mathrm{OH}$ gas in the East core is $\left(4.7_{-3.0}^{+8.0}\right) \times 10^{-9}$ at P2 and $1.5_{-0.9}^{+2.2} \times 10^{-8}$ at P3. As discussed in Section 4.1.2, a possible Galactic counterpart for the East core will be cold and embedded sources such as infrared dark clouds or early-stage YSOs without a hot core region. Thus, the $\mathrm{CH}_{3} \mathrm{OH}$ gas abundances estimated in this work should be compared with those of such Galactic sources. Typical abundances of $\mathrm{CH}_{3} \mathrm{OH}$ gas in Galactic infrared dark clouds are reported to be several $\times 10^{-9}$ (see, e.g., Fig. 3 in Gerner et al. 2014). Similar abundances are reported for Galactic embedded YSOs without hot cores/corinos (e.g., van der Tak et al. 2000; Watanabe et al. 2012; Gerner et al. 2014). Despite a factor of five lower metallicity in the $\mathrm{SMC}$, the $\mathrm{CH}_{3} \mathrm{OH}$ gas abundance at P2 is comparable to those of Galactic counterparts, and that of P3 is marginally higher than the typical Galactic value. If the $\mathrm{CH}_{3} \mathrm{OH}$ gas abundance is simply regulated by the elemental abundances of carbon and oxygen, then the decreased $\mathrm{CH}_{3} \mathrm{OH}$ abundance according to the SMC's metallicity is expected, but this is not observed in the present data. This would indicate an enhanced production or a suppressed destruction of gas-phase $\mathrm{CH}_{3} \mathrm{OH}$ in the East core compared to Galactic similar sources.

The $\mathrm{CH}_{3} \mathrm{OH}$ gas abundance in the East core is comparable to those of two LMC hot cores reported in Sewiło et al. (2018), although the excitation temperature of $\mathrm{CH}_{3} \mathrm{OH}$ in the East core is much lower than those of the two hot cores. Currently, a hot core is not yet detected in the SMC, thus future search for SMC hot cores will be important to further understand low-metallicity organic chemistry in warm and dense gas around a protostar.

\subsubsection{Possible origin of $\mathrm{CH}_{3} \mathrm{OH}$ in the East core}

It is believed that $\mathrm{CH}_{3} \mathrm{OH}$ is mainly formed on dust grains. Hydrogenation of $\mathrm{CO}$ on grain surfaces or energetic processes by photolysis/radiolysis of ice mantles are believed to be responsible for the formation of solid $\mathrm{CH}_{3} \mathrm{OH}$ (e.g., Gerakines et al. 2001; Watanabe et al. 2007). The gas-phase 

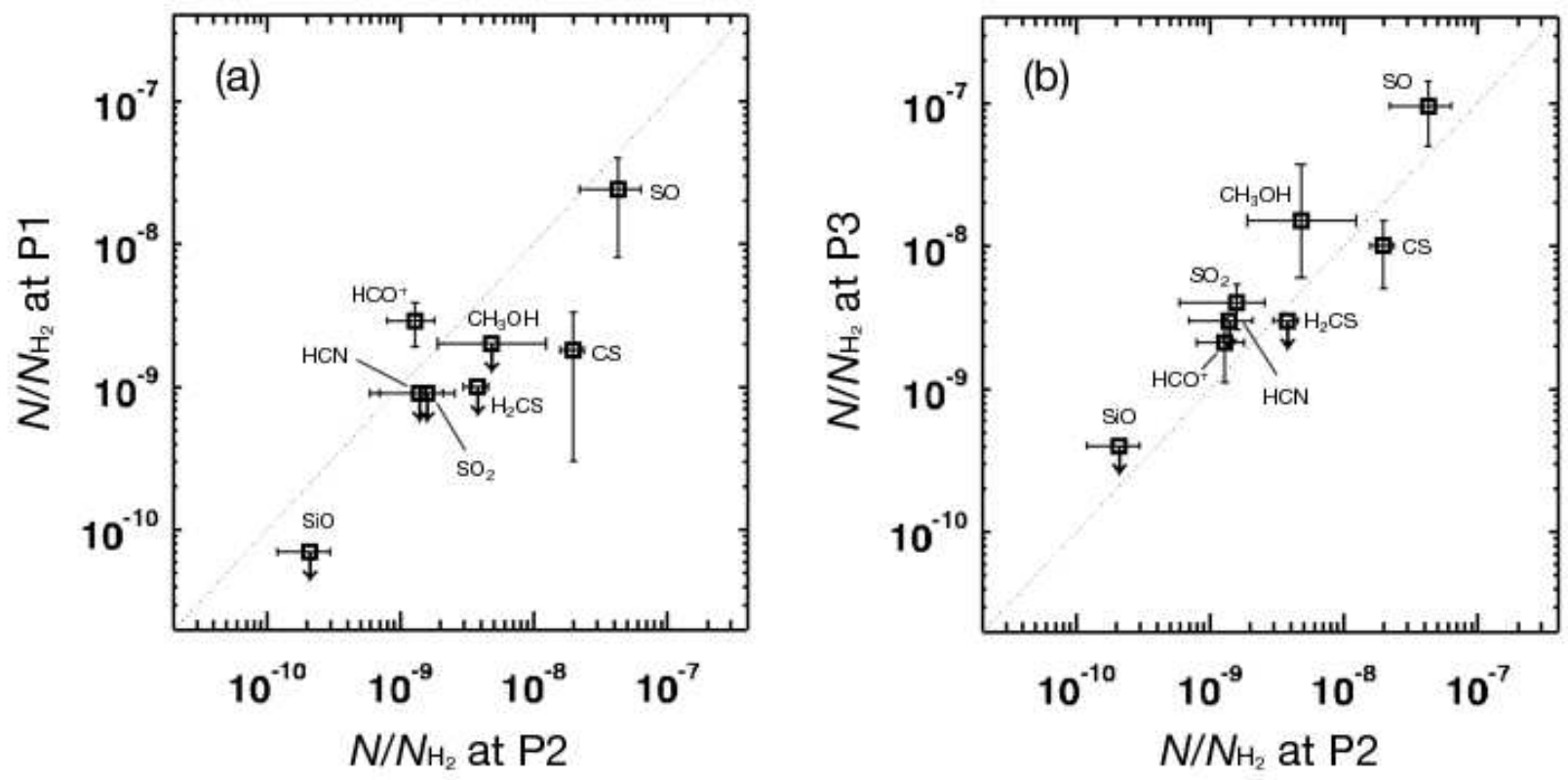

Figure 8. Comparisons of molecular gas abundances at different positions; (a) P1 vs. P2 and (b) P3 vs. P2. Upper and lower limits are indicated by arrows. The dotted lines represent an abundance ratio of 1 . Data of $c-\mathrm{C}_{3} \mathrm{H}_{2}$ are not plotted since it is not detected at all positions. See Section 4.2 for a detailed discussion.

$\mathrm{CH}_{3} \mathrm{OH}$ is thus produced by desorption of solid $\mathrm{CH}_{3} \mathrm{OH}$ from ices.

The desorption mechanisms include (i) sublimation by heating, (ii) photodesorption by UV photons, (iii) sputtering by shocks (e.g., Aota et al. 2015), and (iv) reactive desorption $^{2}$ (e.g., Garrod et al. 2007). The low gas temperature at P2 and P3 suggests that (i) would be less dominant in the East core, since the sublimation temperature of the $\mathrm{CH}_{3} \mathrm{OH}$ ice is higher than $\sim 80 \mathrm{~K}$ (e.g., Tielens 2005). A major source of the UV radiation in the East core would be the cosmic-rayinduced UV photons owing to the high $A_{V}$. However, the average cosmic-ray density in the SMC is reported to be lower than the solar neighborhood by a factor of $\sim 7$ (Abdo et al. 2010). This may lower the efficiency of (ii) compared to the Galactic dense clouds. Note that P3 is located close to the edge of the East core and that the estimated column density and extinction are lower than those at P1 and P2. Thus, the photodesorption of $\mathrm{CH}_{3} \mathrm{OH}$ from ices could be enhanced at P3 compared to P1 and P2 owing to the less attenuated interstellar radiation field. Such a physical condition similar to photodissociation regions may contribute to the high abundance of $\mathrm{CH}_{3} \mathrm{OH}$ gas at $\mathrm{P} 3$.

A common shock tracer, $\mathrm{SiO}$, is marginally detected at $\mathrm{P} 2$, and its line width is possibly broader than those of other emission lines observed in the East core. This suggests a pos-

\footnotetext{
${ }^{2}$ Desorption triggered by liberation of reaction energy
}

sible contribution of the mechanism (iii) to the production of gas-phase $\mathrm{CH}_{3} \mathrm{OH}$. The energy source(s) responsible for triggering the shock chemistry are unknown; possible candidates are outflows from the high-mass YSO located at P1 or outflows from hidden embedded $\mathrm{YSO}(\mathrm{s})$ in the East core. Given the small velocity difference between the East core and the YSO core ( $\sim \mathrm{km} / \mathrm{s}$, see Fig. 5), the collision between the two cores and subsequent shock sputtering of ice mantles are less likely for the production of $\mathrm{CH}_{3} \mathrm{OH}$ gas in the present source (see shock sputtering models in Aota et al. 2015; Miura et al. 2017). The presence of the dynamically continuous bridge-like structure seen in the continuum, CS, and SO lines would support the physical interaction between the YSO core and the East core (Section 3.2). Further observations of intense shock/outflow tracers will help test the contribution of the mechanism (iii). Finally, the mechanism (iv) will be also possible, but its relative contribution should be carefully evaluated with the aid of astrochemical models.

Note that the $\mathrm{CH}_{3} \mathrm{OH}$ ice absorption band at $3.53 \mu \mathrm{m}$ is not detected in the infrared spectrum of the YSO at P1. However, this does not necessarily mean the absence of solid $\mathrm{CH}_{3} \mathrm{OH}$ in the YSO core, because the spectral resolution and sensitivity of the present infrared data are not sufficient to detect a weak absorption band of the $\mathrm{CH}_{3} \mathrm{OH}$ ice.

\subsubsection{Comparison with observations of the LMC and the relevant chemical model}

In the LMC, a low abundance of $\mathrm{CH}_{3} \mathrm{OH}$, compared to similar Galactic objects, is suggested for ices around em- 
bedded high-mass YSOs (Seale et al. 2011; Shimonishi et al. 2016a), warm and dense gas around a hot molecular core (Shimonishi et al. 2016b), and cold diffuse gas in molecular clouds (Nishimura et al. 2016b). The low $\mathrm{CH}_{3} \mathrm{OH}$ abundance is also suggested for molecular clouds in another nearby low-metallicity galaxy, IC10 (Nishimura et al. 2016a). Shimonishi et al. (2016a) proposed the warm ice chemistry hypothesis to interpret the low abundance of $\mathrm{CH}_{3} \mathrm{OH}$ in the LMC. The hypothesis argues that warm dust temperatures inhibit the hydrogenation of $\mathrm{CO}$ in $\mathrm{LMC}$ dense clouds, which contributes to the low $\mathrm{CH}_{3} \mathrm{OH}$ abundances in both solid and gas phase. Astrochemical simulations on gas-ice chemistry dedicated to the LMC environment quantitatively characterize such temperature effects (Acharyya \& Herbst 2015; Pauly \& Garrod 2018). On the other hand, Sewiło et al. (2018) recently detected $\mathrm{CH}_{3} \mathrm{OH}$ gas and its possible daughter species $\left(\mathrm{CH}_{3} \mathrm{OCH}_{3}\right.$ and $\mathrm{CH}_{3} \mathrm{OCHO}$ ) from two hot cores in the LMC. They argue that abundances of these molecular species simply scale with the metallicity of the LMC. The results would suggest the large chemical variation among star-forming cores in the LMC and that the suppression of the $\mathrm{CH}_{3} \mathrm{OH}$ production due to warm ice chemistry does not always dominate the organic chemistry at low metallicity.

In the SMC, the metallicity is even lower than the LMC, and the interstellar radiation field is expected to be less attenuated. This would possibly raise dust temperatures in dense clouds, and the $\mathrm{CH}_{3} \mathrm{OH}$ production could be suppressed if the dust temperature has a dominant effect on grain surface chemistry. However, the $\mathrm{CH}_{3} \mathrm{OH}$ gas abundance in the East core is comparable with those of similar Galactic objects as shown in Section 4.3.1. A decrease of the $\mathrm{CH}_{3} \mathrm{OH}$ abundance, as expected from the inhibition of $\mathrm{CO}$ hydrogenation at elevated dust temperatures, is not seen in the present SMC source.

Shimonishi et al. (2016a) argue that a high $\mathrm{CO}_{2} / \mathrm{H}_{2} \mathrm{O}$ ice column density ratio is another observational indicator of warm ice chemistry, because mobile $\mathrm{CO}$ on warm dust surfaces would efficiently react with $\mathrm{OH}$ to form $\mathrm{CO}_{2}$. Previous observations reported that $\mathrm{CO}_{2} / \mathrm{H}_{2} \mathrm{O}$ ice ratios are about a factor of two larger in envelopes of high-mass YSOs in the LMC ( 30\%, Shimonishi et al. 2008, 2010, 2016a; Oliveira et al. 2009; Seale et al. 2011) as compared with those in Galactic high-mass YSOs $(\sim 17 \%$, Gerakines et al. 1999). The $\mathrm{CO}_{2} / \mathrm{H}_{2} \mathrm{O}$ ice ratio of the high-mass $\mathrm{YSO}$ at $\mathrm{P} 1$ is estimated to be $7_{-3}^{+4} \%$ (Section 4.2), which is lower than the typical ratios for Galactic and LMC high-mass YSOs. Ice abundances in $\mathrm{P} 2 / \mathrm{P} 3$ are currently unknown, but at least in $\mathrm{P} 1$, the observations suggest that the enhanced $\mathrm{CO}_{2}$ ice abundance as observed in LMC YSOs is not clearly seen. The lack of the $\mathrm{CO}_{2} / \mathrm{H}_{2} \mathrm{O}$ ratio enhancement in the SMC is also suggested for a larger sample of embedded high- mass YSOs in the SMC (Oliveira et al. 2013). Oliveira et al. (2011) state that enhanced photodesorption of $\mathrm{H}_{2} \mathrm{O}$ ice in the outer envelope of YSOs can explain the observed characteristics of the $\mathrm{CO}_{2} / \mathrm{H}_{2} \mathrm{O}$ ratios in the LMC/SMC, rather than the grain temperature effect mentioned above.

The above discussions suggest that the chemical properties of dense cores in IRAS 01042-7215 show different characteristics compared with previously observed LMC sources, although statistical properties of dense cloud chemistry at the LMC's and SMC's metallicity are yet to be investigated.

Numerical simulations of gas-ice chemistry dedicated to the SMC and further metal-poor environments are presented in the literature (Acharyya \& Herbst 2016; Pauly \& Garrod 2018; Ceccarelli et al. 2018). Acharyya \& Herbst (2016) show that low grain temperatures (lower than at least 20 $\mathrm{K})$ and well-shielded conditions $\left(A_{V}\right.$ larger than at least 5 mag) are necessary to attain a $\mathrm{CH}_{3} \mathrm{OH}$ gas abundance higher than $10^{-9}$ in SMC dense clouds (see their Fig. 3). The present observational results suggest that a cold, dense, and well-shielded region that is necessary for the production of $\mathrm{CH}_{3} \mathrm{OH}$ actually exists even at the decreased metallicity of the SMC. Interestingly, the astrochemical simulations in Pauly \& Garrod (2018) suggest the enhancement of the $\mathrm{CH}_{3} \mathrm{OH}$ ice abundance relative to the $\mathrm{CO}$ ice in their lowmetallicity models (particularly in their SMC models). According to their explanation, higher grain temperatures decrease the efficiency of the $\mathrm{CO}$ hydrogenation, because more $\mathrm{CO}$ is converted to $\mathrm{CO}_{2}$ and less $\mathrm{CH}_{3} \mathrm{OH}$ is produced, as expected from the warm ice chemistry hypothesis. On the other hand, they also argue that lower metallicities result in slower accretion rates of gaseous heavy elements, which relatively increases the efficiency of the $\mathrm{CO}$ hydrogenation, because this allows $\mathrm{CO}$ to reside on the surface for a longer time for the successive hydrogenation to form $\mathrm{CH}_{3} \mathrm{OH}$, before being embedded in the mantle phase and/or reacting with other heavy surface reactants such as $\mathrm{OH}$. It is likely that the balance between the dust temperature and the abundance of heavy elements is one of the important determinants of the $\mathrm{CO}$ hydrogenation efficiency on grain surfaces. Since neither the $\mathrm{CO}$ ice nor the $\mathrm{CH}_{3} \mathrm{OH}$ ice is detected towards the East core and other embedded sources in the SMC, future highsensitivity observations need to statistically quantify abundances of solid $\mathrm{CO}$ and $\mathrm{CH}_{3} \mathrm{OH}$ in the SMC. This may shed light on the reason for the abundant $\mathrm{CH}_{3} \mathrm{OH}$ gas in the lowmetallicity dense core observed in this work.

We emphasize that it is still unclear whether the chemical composition observed in the East core of IRAS 010427215 represents common characteristics of cold and dense molecular clouds in metal-poor environments. Theoretical studies argue that chemical compositions of dense molecular clouds show a complex dependence on various local environmental factors, notably for species that are mainly pro- 
duced on grain surfaces such as $\mathrm{CH}_{3} \mathrm{OH}$ (Acharyya \& Herbst 2015, 2016; Pauly \& Garrod 2018). It may be possible that patchy distributions of the interstellar medium in an irregular galaxy cause localized and variable radiation environments, which can lead to diverse physical and chemical conditions of molecular clouds compared to our Galaxy. We obviously need to increase the relevant astrochemical data in future observations.

\subsection{Tentative Detection of HDS}

An unidentified line is detected at the rest-frame frequency of $244.5556 \mathrm{GHz}$ (assuming $V_{\mathrm{LSR}}=192.0 \mathrm{~km} \mathrm{~s}^{-1}$ ) in the spectrum of Position 2. The spatial distribution of the emission is similar to those of $\mathrm{C}^{33} \mathrm{~S}, \mathrm{H}_{2} \mathrm{CS}$, and $\mathrm{SiO}$. The line width is consistent with the other lines detected in the East core.

A possible candidate for the line is $\operatorname{HDS}\left(1_{0,1}-0_{0,0}\right)$, which is located at $244.55558 \mathrm{GHz}$ and has the upper-state energy of $11.7 \mathrm{~K}$ according to the CDMS catalogue. The low upperstate energy suggests that this transition can be excited even in the low-temperature condition at P2. If the line is due to HDS $\left(1_{01}-0_{00}\right)$, then the observed line strength corresponds to $N(\mathrm{HDS})=(5 \pm 1.3) \times 10^{13} \mathrm{~cm}^{-2}$ and $N(\mathrm{HDS}) / N_{\mathrm{H}_{2}}$ $=8 \times 10^{-10}$ (assuming $T_{\text {rot }}=12 \mathrm{~K}$ ). Abundances of HDS in Galactic star-forming cores are typically in the range of $10^{-11}-10^{-10}$ according to Vastel et al. (2003). The estimated HDS abundance at the East core is comparable to typical Galactic values despite the lower elemental abundances in the SMC. Further observations of other transitions and related molecular species are highly required to understand deuterium chemistry in low-metallicity environments.

\section{SUMMARY}

We report the results of $0.1 \mathrm{pc}$ scale submillimeter observations towards a high-mass young stellar object (IRAS 010427215 ) in the Small Magellanic Cloud with ALMA. Complementary infrared data obtained with AKARI and the Gemini South telescope are also presented. The following conclusions are obtained in this work:

1. Emission lines of $\mathrm{CS}, \mathrm{C}^{33} \mathrm{~S}, \mathrm{H}_{2} \mathrm{CS}, \mathrm{SO}, \mathrm{SO}_{2}, \mathrm{CH}_{3} \mathrm{OH}$, $\mathrm{H}^{13} \mathrm{CO}^{+}, \mathrm{H}^{13} \mathrm{CN}, \mathrm{SiO}$, and dust continuum are detected. Among these species, $\mathrm{CH}_{3} \mathrm{OH}, \mathrm{SO}_{2}, \mathrm{H}_{2} \mathrm{CS}$, $\mathrm{C}^{33} \mathrm{~S}$, and $\mathrm{H}^{13} \mathrm{CN}$ are for the first time detected in the SMC. Tentative detection of HDS is also reported.

2. The target source is spatially resolved into two dense molecular cloud cores, named the YSO core and the East core. The YSO core is very dense $\left(n_{\mathrm{H}_{2}} \sim 10^{6}\right.$ $\left.\mathrm{cm}^{-3}\right)$ and well shielded $\left(A_{V} \sim 10-30 \mathrm{mag}\right)$, and is associated with an embedded high-mass YSO. The East core is also dense $\left(n_{\mathrm{H}_{2}} \sim 10^{5} \mathrm{~cm}^{-3}\right)$ and shielded $\left(A_{V}\right.$ $\sim 4-10 \mathrm{mag}$ ), but is not associated with an infrared source. A gas temperature in the East core is estimated to be $\sim 10 \mathrm{~K}$ based on the rotation analysis of $\mathrm{CH}_{3} \mathrm{OH}$ lines. A possible Galactic counterpart for the East core would be a massive starless core, an embedded highmass YSO(s) before the emergence of infrared emission, or a cluster of low-mass embedded YSOs.

3. The above two dense cores, which are separated by $\sim 0.3 \mathrm{pc}$, show different chemical characteristics. The chemical composition of the YSO core is characterized by the low abundance of molecular gas and the presence of ices. In contrast, the East core is characterized by relatively rich molecular lines, and the species except for $\mathrm{CS}$, SO, and $\mathrm{H}^{13} \mathrm{CO}^{+}$are detected only in the East core. Ices are not detected toward the East core in the current data owing to the lack of a bright infrared continuum source. We speculate that the effect of shock chemistry and/or the different degree of the adsorption of gas-phase species onto dust may contribute to the chemical differentiation.

4. The fractional abundance of $\mathrm{CH}_{3} \mathrm{OH}$ gas in the East core is estimated to be $(0.5-1.5) \times 10^{-8}$, which is comparable with or marginally higher than those of similar cold sources in our Galaxy despite a factor of five lower metallicity in the SMC. This would indicate an enhanced production or a suppressed destruction of gas-phase $\mathrm{CH}_{3} \mathrm{OH}$ in the present SMC source compared to Galactic analogues. Although it is still unclear whether the observed source represents common characteristics of cold and dense molecular gas in the SMC, this work provides observational evidence that an organic molecule like $\mathrm{CH}_{3} \mathrm{OH}$, which is largely formed on grain surfaces, can be produced even in a significantly lower metallicity environment compared to the solar neighborhood. A possible origin of cold $\mathrm{CH}_{3} \mathrm{OH}$ gas in the East core is discussed.

This paper makes use of the following ALMA data: ADS/JAO.ALMA\#2016.1.00394.S. ALMA is a partnership of ESO (representing its member states), NSF (USA) and NINS (Japan), together with NRC (Canada) and NSC and ASIAA (Taiwan) and KASI (Republic of Korea), in cooperation with the Republic of Chile. The Joint ALMA Observatory is operated by ESO, AUI/NRAO and NAOJ. This work has made extensive use of the Cologne Database for Molecular Spectroscopy. This work uses data based on observations with AKARI, a JAXA project with the participation of ESA. This work also uses data obtained at the Gemini South telescope as a part of the time exchange program between the Gemini and Subaru telescopes (Program ID: S10B-120). We also use data obtained by the Two Micron All Sky Survey 
project, NASA's Spitzer Space Telescope, and ESA's Herschel Space Observatory. We are grateful to all the members who contributed to these projects. This work is supported by a Grant-in-Aid from the Japan Society for the Promotion of Science (15K17612). T. Shimonishi was supported by the ALMA Japan Research Grant of NAOJ Chile Observatory, NAOJ-ALMA-183. Y.N. was supported by NAOJ ALMA Scientific Research grant Number 2017-06B. Finally, we would like to thank an anonymous referee for insightful comments, which substantially improved this paper.

\section{REFERENCES}

Abdo, A. A., Ackermann, M., Ajello, M., et al. 2010, A\&A, 523, A46

Acharyya, K., \& Herbst, E. 2015, ApJ, 812, 142

—. 2016, ApJ, 822, 105

Aguirre, J. E., Bezaire, J. J., Cheng, E. S., et al. 2003, ApJ, 596, 273

Aota, T., Inoue, T., \& Aikawa, Y. 2015, ApJ, 799, 141

Ceccarelli, C., Viti, S., Balucani, N., \& Taquet, V. 2018, MNRAS, 476, 1371

Chin, Y.-N., Henkel, C., Millar, T. J., Whiteoak, J. B., \&

Marx-Zimmer, M. 1998, A\&A, 330, 901

Chin, Y.-N., Henkel, C., Whiteoak, J. B., et al. 1997, A\&A, 317, 548

Cox, A. N. 2000, Allen's astrophysical quantities (Springer)

Dufour, R. J. 1984, in IAU Symposium, Vol. 108, Structure and Evolution of the Magellanic Clouds, ed. S. van den Bergh \& K. S. D. de Boer, 353-360

Ellingsen, S. P., Breen, S. L., Caswell, J. L., Quinn, L. J., \& Fuller, G. A. 2010, MNRAS, 404, 779

Garrod, R. T., Wakelam, V., \& Herbst, E. 2007, A\&A, 467, 1103

Gerakines, P. A., Moore, M. H., \& Hudson, R. L. 2001, J. Geophys. Res., 106, 33381

Gerakines, P. A., Whittet, D. C. B., Ehrenfreund, P., et al. 1999, ApJ, 522, 357

Gerner, T., Beuther, H., Semenov, D., et al. 2014, A\&A, 563, A97

Goldsmith, P. F., \& Langer, W. D. 1999, ApJ, 517, 209

Graczyk, D., Pietrzyński, G., Thompson, I. B., et al. 2014, ApJ, 780,59

Green, J. A., Caswell, J. L., Fuller, G. A., et al. 2008, MNRAS, 385,948

Heikkilä, A., Johansson, L. E. B., \& Olofsson, H. 1999, A\&A, 344, 817

Israel, F. P., Johansson, L. E. B., Lequeux, J., et al. 1993, A\&A, 276, 25

Kemper, F., Woods, P. M., Antoniou, V., et al. 2010, PASP, 122, 683

Leroy, A., Bolatto, A., Stanimirovic, S., et al. 2007, ApJ, 658, 1027

Martin, N., Maurice, E., \& Lequeux, J. 1989, A\&A, 215, 219

Meixner, M., Gordon, K. D., Indebetouw, R., et al. 2006, AJ, 132, 2268

Meixner, M., Panuzzo, P., Roman-Duval, J., et al. 2013, AJ, 146, 62
Miura, H., Yamamoto, T., Nomura, H., et al. 2017, ApJ, 839, 47

Müller, H. S. P., Schlöder, F., Stutzki, J., \& Winnewisser, G. 2005, Journal of Molecular Structure, 742, 215

Müller, H. S. P., Thorwirth, S., Roth, D. A., \& Winnewisser, G. 2001, A\&A, 370, L49

Murakami, H., Baba, H., Barthel, P., et al. 2007, PASJ, 59, 369

Muraoka, K., Homma, A., Onishi, T., et al. 2017, ApJ, 844, 98

Nishimura, Y., Shimonishi, T., Watanabe, Y., et al. 2016a, ApJ, 829, 94

-. 2016b, ApJ, 818, 161

Oliveira, J. M., van Loon, J. T., Chen, C., et al. 2009, ApJ, 707, 1269

Oliveira, J. M., van Loon, J. T., Sloan, G. C., et al. 2011, MNRAS, 411, L36

-. 2013, MNRAS, 428, 3001

Onaka, T., Matsuhara, H., Wada, T., et al. 2007, PASJ, 59, 401

Ossenkopf, V., \& Henning, T. 1994, A\&A, 291, 943

Pauly, T., \& Garrod, R. T. 2018, ApJ, 854, 13

Roman-Duval, J., Bot, C., Chastenet, J., \& Gordon, K. 2017, ApJ, 841,72

Ruffle, P. M. E., Kemper, F., Jones, O. C., et al. 2015, MNRAS, 451,3504

Russell, S. C., \& Dopita, M. A. 1992, ApJ, 384, 508

Seale, J. P., Looney, L. W., Chen, C.-H. R., Chu, Y.-H., \& Gruendl, R. A. 2011, ApJ, 727, 36

Sewiło, M., Indebetouw, R., Charnley, S. B., et al. 2018, ApJL, 853, L19

Shimonishi, T. 2012, PhD thesis, The University of Tokyo

Shimonishi, T., Dartois, E., Onaka, T., \& Boulanger, F. 2016a, A\&A, 585, A107

Shimonishi, T., Onaka, T., Kato, D., et al. 2008, ApJL, 686, L99

-. 2010, A\&A, 514, A12

Shimonishi, T., Onaka, T., Kawamura, A., \& Aikawa, Y. 2016b, ApJ, 827, 72

Skrutskie, M. F., Cutri, R. M., Stiening, R., et al. 2006, AJ, 131, 1163

Soma, T., Sakai, N., Watanabe, Y., \& Yamamoto, S. 2015, ApJ, 802,74

Sutton, E. C., Peng, R., Danchi, W. C., et al. 1995, ApJS, 97, 455

Tafalla, M., Santiago-García, J., Myers, P. C., et al. 2006, A\&A, 455,577 
Tielens, A. G. G. M. 2005, The Physics and Chemistry of the Interstellar Medium

van der Tak, F. F. S., van Dishoeck, E. F., \& Caselli, P. 2000, A\&A, 361,327

van Dishoeck, E. F., Blake, G. A., Jansen, D. J., \& Groesbeck,

T. D. 1995, ApJ, 447, 760

van Loon, J. T., Cohen, M., Oliveira, J. M., et al. 2008, A\&A, 487, 1055

van Loon, J. T., Oliveira, J. M., Gordon, K. D., Sloan, G. C., \& Engelbracht, C. W. 2010, AJ, 139, 1553
Vastel, C., Phillips, T. G., Ceccarelli, C., \& Pearson, J. 2003, ApJL, 593, L97

Venn, K. A. 1999, ApJ, 518, 405

Wang, M., Chin, Y.-N., Henkel, C., Whiteoak, J. B., \& Cunningham, M. 2009, ApJ, 690, 580

Ward, J. L., Oliveira, J. M., van Loon, J. T., \& Sewiło, M. 2017, MNRAS, 464, 1512

Watanabe, N., Mouri, O., Nagaoka, A., et al. 2007, ApJ, 668, 1001

Watanabe, Y., Sakai, N., Lindberg, J. E., et al. 2012, ApJ, 745, 126

Whittet, D. C. B., Gerakines, P. A., Hough, J. H., \& Shenoy, S. S. 2001, ApJ, 547, 872 